\title{
LESS-EXPENSIVE VALUATION AND RESERVING OF LONG-DATED VARIABLE ANNUITIES WHEN INTEREST RATES AND MORTALITY RATES ARE STOCHASTIC
}

\author{
KEVIN FERGUSSON
}

\begin{abstract}
Variable annuities are products offered by pension funds and life offices that provide periodic future payments to the investor, and often have ancillary benefits that guarantee survival benefits or sums insured on death. This paper extends the benchmark approach to value and hedge long-dated variable annuities using a combination of cash, bonds and equities under a variety of market models, allowing for dependence between financial and insurance markets. Under a simplified case of independence, the results show that when the discounted index is modelled as a time-transformed squared Bessel process less-expensive valuation and reserving is achieved regardless of the short rate model or the mortality model.
\end{abstract}

\section{INTRODUCTION}

Wealth management companies, pension funds and insurers offer long-term savings products such as variable annuities (VAs) to individuals planning for retirement. Such institutions are interested in managing their risk exposure either through reinsurance, derivative markets, or in-house hedging or reserving programmes. Most of the principles for valuation and computing risk sensitivities are closely related to the classical risk-neutral valuation approach. In the low interest rate environment that has been prevailing in recent years variable annuity products appear to be rather expensive and questions arise whether some insurers can in future match assets with liabilities when valuing and managing variable annuities in a traditional manner.

The benchmark approach (BA) of Platen and Heath [2006] allows less-expensive valuation and hedging of long-dated products without requiring the existence of an equivalent risk-neutral probability measure. Within the current paper we demonstrate how to apply this approach to less-expensive valuation and hedging variable annuities when compared to the classical approach.

The literature on valuation and hedging variable annuities is vast and includes, for example, Milevsky and Posner [2001], Boyle and Hardy [2003], Ulm [2008], Bauer et al. [2008], Marquardt et al. [2008], van Haastrecht et al. [2009] and Bacinello et al. [2011]. Typically a variable annuity issued by an insurance company has various product features such as death benefit protection options, living benefit protection options and lifetime income options. In this article we demonstrate

Date: February 18, 2020.

1991 Mathematics Subject Classification. Primary 62P05; Secondary 60G35, 62P20.

Key words and phrases. Variable annuities, growth optimal portfolio, benchmark approach, long-dated equity index options, minimal market model, stochastic short rate, stochastic mortality rates. 
the application of the BA with variable annuities having living benefit and death benefit protections, that is, the benefit is guaranteed if the investor survives and dies, respectively. This guarantee is akin to a long-dated equity put option with a survival or death condition and, therefore, we must employ models for the mortality, equity index and interest rate associated with the variable annuity.

Some work that has been published on valuation and hedging of long-dated equity derivatives includes, for example, Hulley and Platen [2008] who consider equity index options on the S\&P500 total return index for the case of deterministic interest rates. Hedging of long-dated equity index options, when interest rates are stochastic, has been studied in Fergusson and Platen [2015a] and, more recently in Zhu et al. [2018]. The focus of the article of Zhu et al. [2018] is on interest rate risk inherent in crediting rates for cash balance pension plans, employing the single-factor and two-factor Hull-White models. The significant contribution of this paper is the application of the benchmark approach to valuing VAs and the incorporation of a dependence structure for the stock market, interest rates and mortality rates. While closed-form solutions are difficult to obtain in general for dependent components, our model of dependence is tractable, permitting explicit closed-form formulae for integral transforms of values of VAs and enabling backtests of hedging and reserving strategies to be performed.

In this article we demonstrate less-expensive valuation and hedging of long-dated variable annuities under various market models specified by stochastic differential equations (SDEs) of the short rate, the discounted stock index and the mortality rate. In Section 2 the VA product having death, surrender and accumulation benefits is described. In Section 3 we give general SDEs of the short rate, the discounted stock index and the force of mortality, allowing for dependence. The parameter values of each model are fitted. In Section 4.1 the surrender benefit is valued, this being a version of the basic mortality security, the survivor zero-coupon bond or pure endowment. In Section 4.2 valuation formulae for the death benefits and accumulation benefit, each having embedded guarantees, are supplied. These two sections provide the basis for the valuation formulae for variable annuities, having guaranteed accumulation benefits and guaranteed death benefits, in Section 4.3. In Section 5 the reserving and hedging method is described and, in the particular instances where each of the short rate, discounted GOP and mortality rate is driven by independent Wiener processes, backtests of reserving for a variable annuity are supplied. Also, in respect of a put option, whose strike price is an exponential function of time multiplied by the spot price, which is the type of option embedded in the VA, the 99-th percentile costs of hedging are graphically shown over varying times to expiry and over each of the considered models. In Section 6 we conclude. For the interested reader, Appendix A gives some background to the valuation of contingent claims under the benchmark approach. Appendix B provides graphs of simulations under the fitted models versus actual behaviours of corresponding variables. Appendix C provides graphs of simulated reserves and policy values when the assumptions implicit in the VA product are varied.

\section{Description of the VA Product}

We consider a variable annuity product, having as policy riders a guaranteed death benefit, a surrender value and a guaranteed accumulation benefit that is also capped. We let $t_{0}$ denote the time at which the policy is purchased for one dollar 
and let $t_{1}<t_{2}<\ldots<t_{n}$ denote the $n$ anniversary times at which either death, surrender or accumulation benefits are paid. A unit premium paid for the policy at time $t_{0}$ is invested immediately in an investment account, which is used to pay benefits, and from which a management fee is continuously deducted at the rate $\xi$ per annum. We assume $\xi=0.03$ in this paper, which is consistent with the level of management fees quoted in Haithcock [2013], for example. Simulations of the VA reserves and policy values over the life of the policy portfolio for alternative values of $\xi$ are given in Appendix C.

\section{Death Benefit}

In respect of the death benefit, the guarantee at the end of year of death is a roll-up of the initial premium, continuously compounded at a rate $g$ per annum, which we set to 0.03 . This choice of $g$ is selected as being commensurate with the average historical US cash deposit rate. Simulations of the VA reserves and policy values over the life of the policy portfolio for alternative values of $g$ are given in Appendix C. Therefore, for a policyholder aged $x+t$ at time $t$ and who dies at time $\tau_{x+t}(t)>t$, where $\tau_{x+t}(t) \in\left[t_{i-1}, t_{i}\right)$, the payoff on death will be

$$
H_{t_{i}}^{(D)}=F_{t_{i}}+\left(K_{t_{i}}-F_{t_{i}}\right)^{+},
$$

where $K_{t_{i}}=\exp \left\{g\left(t_{i}-t_{0}\right)\right\}$ denotes the strike price, or guaranteed death benefit, of the variable annuity at time $t_{i}$, the net fund value $F_{t_{i}}$ is

$$
F_{t_{i}}=\frac{S_{t_{i}}}{S_{t_{0}}} \exp \left\{-\xi\left(t_{i}-t_{0}\right)\right\},
$$

and $S_{t}$ denotes the value at time $t$ of the equity market index, inclusive of dividends, as described in Section 3.

\section{Surrender Value}

To accommodate lapses in the policy, we specify a surrender value equal to a roll-up of the initial premium, continuously compounded at a rate $g / 2$ per annum. Other than being less than the roll-up rate $g$ for the guarantee, this choice of $g / 2$ is arbitrary. Simulations of the VA reserves and policy values over the life of the policy portfolio for alternative values of multiplicative factor $1 / 2$ applied to the roll-up rate in respect of the surrender value are given in Appendix C.

Therefore, for a policyholder aged $x+t$ at time $t$ and who lapses at time $\tau_{x+t}^{(L)}(t)>$ $t$, where $\tau_{x+t}^{(L)}(t) \in\left[t_{i-1}, t_{i}\right)$, the payoff of the VA will be

$$
H_{t_{i}}^{(L)}=\sqrt{K_{t_{i}}} \text {. }
$$

The lapse rate is defined as the proportion of policies which are surrendered in a given year. We assume, for simplicity, an annual lapse rate of $\rho=0.03$ among those lives who do not die during the year. In Marquardt et al. [2008], a more realistic lapse rate schedule is assumed. In practice, insurers model the lapse behaviour of policyholders with reference to the moneyness of the embedded guarantees; see Escobar et al. [2016] and Hartman [2018]. While there is scope to enhance the lapse rate assumption in this paper, we maintain our constant lapse rate assumption with the aim of illustrating the advantages of the benchmark approach in Section 5.3.

\section{Accumulation Benefit}

In respect of the accumulation benefit, the guarantee at the maturity of the policy is a roll-up of the initial premium, continuously compounded at the same annual rate $g$ used for the death benefit guarantee. Additionally, the accumulation 
benefit at maturity is capped at the roll-up of the initial premium continuously compounded at the rate $3 g$ per annum.

Therefore, for a policyholder aged $x+t$ at time $t$ and who survives to time $t_{n}$, the payoff of the guaranteed accumulation benefit with cap is

$$
H_{t_{n}}^{(A)}=F_{t_{n}}+\left(K_{t_{n}}-F_{t_{n}}\right) I_{F_{t_{n}}<K_{t_{n}}}-\left(F_{t_{n}}-K_{t_{n}}^{3}\right) I_{F_{t_{n}}>K_{t_{n}}^{3}},
$$

which can be rewritten as

$$
H_{t_{n}}^{(A)}= \begin{cases}K_{t_{n}}, & F_{t_{n}}<K_{t_{n}} \\ F_{t_{n}}, & K_{t_{n}}<F_{t_{n}}<K_{t_{n}}^{3} \\ K_{t_{n}}^{3}, & F_{t_{n}}>K_{t_{n}}^{3}\end{cases}
$$

Remark 1. Certainly by taking $\xi=g=\rho=0.03$ we have fees of 0.03 p.a. deducted from the investment account, the guarantee accumulating at a rate of 0.03 p.a. and the inforce policies decumulating from lapses at a rate of 0.03 p.a. and, in the hypothetical case of all non-lapsed policyholders dying in the $n$-th year, the aggregate of minimum death benefits guaranteed by the insurer at the end of the $n$-th year is the multiple $\exp (0.03 n) \times 0.97^{n}$ of the total of initial premiums received. This multiple is not much smaller than one, e.g. it is 0.9773 for $n=50$ years, so the effect of the guarantee remains an important consideration for the insurer. Bearing in mind that management fees $\xi=0.03$ are deducted from the wealth accounts, this guarantee means that the insurer needs to manage the policyholders' wealth accounts by achieving gross returns in excess of $\xi=0.03$ p.a. to avoid the insurer's liabilities exceeding the assets. Simulations of the VA reserves and policy values over the life of the policy portfolio for alternative values of $\xi, g$ and $\rho$ are given in Appendix $C$.

In a complete market, where there exist traded mortality derivatives such as mortality forwards, survivor forwards and longevity swaps, it would be possible to hedge the mortality risk inherent in the variable annuity, barring any basis risk. However, we assume that such derivatives are not available for hedging and therefore resort to accepting the longevity risk of our mortality model and diversifying away the stochastic mortality risk.

\section{The Modelling Framework}

Our structure of the market in which VAs are valued is an investible savings account, an investible ten-year coupon bond, and an investible diversified stock index, which allows interest rate and equity market risks to be hedged, but which leaves mortality risk unhedgeable and, therefore, the market for VAs incomplete. The ten-year bond is one of the primary assets, as stated in Appendix A.

Typically, valuation of financial contracts employs the no-arbitrage principle and it is important to clarify the types of arbitrage opportunities that are prohibited. In the classical sense, arbitrage, as espoused by Delbaen and Schachermayer [1994], refers to the ability to extract profits with vanishing risk and the authors show that a unique no-arbitrage price for a contingent claim exists when the market is complete and an equivalent risk neutral probability measure exists. On the other hand, strong arbitrage, as espoused by Platen and Heath [2006], refers to the ability to generate, under limited liability, strictly positive wealth from zero initial capital. The benchmark approach makes use of the GOP as the numeraire or benchmark, which renders the benchmarked price process of any positively valued portfolio 
as a supermartingale, thereby prohibiting strong arbitrage, but which may allow classical arbitrage. As a result, in many considered models risk-neutral valuation cannot be applied and, therefore, valuation of a contract is performed via the realworld valuation formula (A.1), which, for a hedgeable security, gives the minimal value of a replicating portfolio, while for a security having nonhedgeable risk, gives the actuarial value or expected present value of claims under the contract. The existence of an equivalent risk-neutral probability measure is not required for the real-world valuation, but agreement is obtained with the risk-neutral valuation when this probability measure exists and the market is complete.

Let $(\Omega, \mathcal{A}, \underline{\mathcal{A}}, P)$ denote the filtered probability space in which we work, where the filtration $\mathcal{A}=\left(\mathcal{A}_{t}\right)_{t>0}$ satisfies the usual conditions, as described in Karatzas and Shreve [1991]. We denote the short rate process by $r=\left\{r_{t}: t \geq 0\right\}$, of which the overnight cash rate is considered a realistic proxy. The savings account is then denoted by $B_{t}=\exp \left(\int_{0}^{t} r_{s} d s\right)$, for every $t \geq 0$.

The central building block of the benchmark approach is the growth optimal portfolio (GOP), denoted by $S_{t}$ at time $t \geq 0$. For the equity market the GOP is well approximated by well diversified total return equity indices such as the S\&P 500 total return index, as demonstrated by Platen and Rendek [2012]. In particular, in Platen [2005] and Platen and Rendek [2012] it is proven that appropriately defined diversified portfolios represent approximate GOPs and therefore a number of commonly used well-diversified stock market indices can be used to approximate the GOP, including but not limited to the following: Standard and Poor's 500 Index and Russell 2000 Index for the US market and the MSCI Growth World Index for global modelling. These indices are listed and their construction is based on the published numbers of shares of their component stocks. The explicit construction of well diversified portfolios based on naive diversification is explained in Platen and Rendek [2012]. The discounted GOP is denoted by $\bar{S}_{t}$, being equal to the value of the GOP denominated in units of the savings account.

As demonstrated in Platen [2001] and summarised in Appendix A, the SDE satisfied by the GOP is

$$
d S_{t}=S_{t}\left(r_{t}+\theta_{t}^{2}\right) d t+S_{t} \theta_{t} d W_{t},
$$

where $\theta=\left\{\theta_{t}: t \geq 0\right\}$ denotes the market price of risk of the GOP, and, therefore, the SDE satisfied by the discounted GOP is

$$
d \bar{S}_{t}=\bar{S}_{t} \theta_{t}^{2} d t+\bar{S}_{t} \theta_{t} d W_{t} .
$$

Here $W=\left\{W_{t}: t \geq 0\right\}$ is a Wiener process adapted to the filtration $\underline{\mathcal{A}}$.

3.1. Discounted GOP Models. The models of the discounted GOP that are considered are the Black-Scholes model, see Black and Scholes [1973], with constant volatility $\theta>0$, where

$$
d \bar{S}_{t}=\bar{S}_{t} \theta^{2} d t+\bar{S}_{t} \theta d W_{t}
$$

and the minimal market model (MMM) described by Platen [2001]

$$
d \bar{S}_{t}=\bar{\alpha}_{t} d t+\sqrt{\bar{\alpha}_{t} \bar{S}_{t}} d W_{t}
$$

where $\bar{\alpha}_{t}$ is specifically chosen as $\bar{\alpha}_{t}=\bar{\alpha}_{0} \exp (\eta t)$, with constant net growth rate $\eta>0$. Here $\theta, \bar{\alpha}_{0}$ and $\eta$ are constants in the respective models. 
Connected with the modelling of the discounted GOP is the normalised GOP, defined as

$$
Y_{t}=\frac{\eta \bar{S}_{t}}{\bar{\alpha}_{t}} .
$$

For the Black-Scholes model, the SDE for $Y$ is

$$
d Y_{t}=\left(\theta^{2}-\eta\right) Y_{t} d t+\theta Y_{t} d W_{t},
$$

which is geometric Brownian motion, whereas for the MMM, the SDE for $Y$ is

$$
d Y_{t}=\eta\left(1-Y_{t}\right) d t+\sqrt{\eta Y_{t}} d W_{t},
$$

which is a square root process having a mean-reverting level of one. In what follows, the normalised GOP is used to incorporate dependence of the short rate and mortality rate on the GOP.

3.2. Short Rate Models. We model the short rate $r$ as a linear combination of a function of the normalised GOP $Y$ and, what we term, the primitive short rate $R$, via

$$
r_{t}=\alpha^{r} f^{r}\left(Y_{t}\right)+R_{t},
$$

where $f^{r}$ is a function, being either $f^{r}(x)=x, f^{r}(x)=\log x$ or $f^{r}(x)=1 / x$, and $\alpha^{r}$ is a constant. Here the process $R$ is independent of the process $Y$.

The primitive short rate is the contribution to the short rate which is not explained by the contribution from the normalised GOP. The economic intuition behind the primitive short rate is that monetary policy in many cases will be influenced by the growth of the economy and the stock market. For example, in boom periods of the stock market, which are typically fuelled by low interest rates, monetary policy may be tightened to temper the market excesses and allow the economy to travel more smoothly. Also, in market lulls, which reflect low economic growth, monetary policy may be loosened to invigorate the economy. Viewed in this way, the primitive short rate avoids the effects of the stock market which influence the short rate.

The primitive short rate models we consider are the deterministic short rate model, where the short rate is assumed to be known as a function of time,

$$
R_{t}=R(t),
$$

the Vasicek short rate model described by Vasicek [1977],

$$
d R_{t}=\kappa\left(\bar{R}-R_{t}\right) d t+\sigma d Z_{t},
$$

where $\bar{R}, \kappa$ and $\sigma$ are positive constants, the Cox-Ingersoll-Ross (CIR) short rate model described by Cox et al. [1985],

$$
d R_{t}=\kappa\left(\bar{R}-R_{t}\right) d t+\sigma \sqrt{R_{t}} d Z_{t},
$$

where $\bar{R}, \kappa$ and $\sigma$ are positive constants such that $2 \bar{R} \kappa / \sigma^{2}>1$, and the $3 / 2$ short rate model first theorised by Platen [1999], and described by Ahn and Gao [1999],

$$
d R_{t}=\left(p R_{t}+q R_{t}^{2}\right) d t+\sigma R_{t}^{3 / 2} d Z_{t},
$$

where $p>0, q$ and $\sigma>0$ are constants such that $q<\sigma^{2} / 2$, and where models are driven by a Wiener process $Z=\left\{Z_{t}: t \geq 0\right\}$ adapted to the filtration $\underline{\mathcal{A}}$ and independent of the Wiener process $W$. 


\begin{tabular}{|l|l|}
\hline Model & Mortality Rate Formula \\
\hline Gompertz & $\mu_{x}=b \exp (c x)$ \\
Makeham & $\mu_{x}=a+b \exp (c x)$ \\
Thiele & $\mu_{x}=b \exp (c x)+f_{0} \exp \left(-f_{1}\left(x-f_{2}\right)^{2}\right)+g \exp (h x)$ \\
Siler & $\mu_{x}=a+b \exp (c x)+g \exp (h x)$ \\
\hline
\end{tabular}

TABLE 1 . Formulae for mortality rates of various models.

3.3. Mortality Models. We model the mortality rate $\mu_{x+t}(t)$, in respect of a life aged $x+t$ at time $t$, as a linear combination of a function of the normalised GOP $Y$, the primitive short rate $R$ and, what we term, the primitive mortality rate $M_{x+t}(t)$, via

$$
\mu_{x+t}(t)=\alpha^{\mu} f^{\mu}\left(Y_{t}\right)+\beta^{\mu} R_{t}+M_{x+t}(t),
$$

where $f^{\mu}$ is either of the functions potentially assumed by $f^{r}$ earlier, and $\alpha^{\mu}$ and $\beta^{\mu}$ are constants.

To capture mortality, let $\ell_{x}$ denote the average number of lives alive at age $x$ among a cohort of $\ell_{0}$ lives, as done in actuarial life tables. An actuarial life table is typically constructed by observing the mortality rates of a population of lives over an investigation period, centred at some specific time $t$. Because mortality rates at all ages change over time, it is necessary to conduct regular mortality investigations to produce new actuarial life tables. Assuming static mortality rates, Gompertz [1825] modelled $\ell_{x}$ as

$$
\ell_{x}=\ell_{0} \exp \left(-b \int_{0}^{x} \exp (c s) d s\right),
$$

for constant parameters $b$ and $c$. This model was enriched by Makeham [1860] by

$$
\ell_{x}=\ell_{0} \exp \left(-a x-b \int_{0}^{x} \exp (c s) d s\right),
$$

through adding an extra growth rate $a$. In Thiele [1871] the Gompertz-Makeham model was even further enhanced by the addition of terms to fit infant mortality and adolescent mortality, where $\ell_{x}$ is modelled as

$$
\ell_{x}=\ell_{0} \exp \left(-b \int_{0}^{x} \exp (c s) d s-f_{0} \int_{0}^{x} \exp \left(-f_{1}\left(s-f_{2}\right)^{2}\right) d s-g \int_{0}^{x} \exp (h s) d s\right),
$$

with constant parameters $f_{0}, f_{1}, f_{2}, g$ and $h$. Also, in Siler [1979] and Siler [1983] a simpler enhancement to the Gompertz-Makeham model involved only the addition of a term to fit infant mortality where $\ell_{x}$ is modelled as

$$
\ell_{x}=\ell_{0} \exp \left(-a x-b \int_{0}^{x} \exp (c s) d s-g \int_{0}^{x} \exp (h s) d s\right),
$$

with constant parameters $g$ and $h$.

The logarithmic mortality rate at age $x$ can then be denoted by $\mu_{x}=-\frac{d}{d x} \log \ell_{x}$, the formulae of which are shown for the previous mortality models in Table 1. To allow for changes in mortality rates over time, in Lee and Carter [1992] the model

$$
\log m_{x}(t)=a_{x}+b_{x} k_{t}+\epsilon_{x, t}
$$


was proposed, where $t \in\{1,2, \ldots, T\}, m_{x}(t)$ is the central mortality rate of a life aged $x$ at time $t, \epsilon_{x, t}$ is the residual, $a_{x}=\frac{1}{T} \sum_{t} m_{x}(t), b_{x}$ is a rate of mortality improvement at age $x$ such that $\sum_{x} b_{x}=1$ and $k_{t}$ is the mortality trend which satisfies $\sum_{t} k_{t}=0$. The least squares solution to (3.16) can be found using singular value decomposition on the matrix $M=\left(m_{x}(t)-a_{x}\right)_{x, t}$. We can modify (3.16), allowing $\log \mu_{x}(t)$ to be modelled as the random process

$$
\log \mu_{x}(t)=a_{x}+b_{x}\left(t+\gamma X_{t}\right),
$$

where $X=\left\{X_{t}: t \geq 0\right\}$ is a Brownian motion and $a_{x}=a_{0}+a_{1} x$ and $b_{x}=$ $b_{0}+b_{1} x$ are linear functions of $x$, for constants $a_{0}, a_{1}, b_{0}$ and $b_{1}$. Without any time dependence, (3.17) becomes Gompertz' model. Applying Itô's Lemma to (3.17), the SDE for $\mu_{x}(t)$ is

$$
d \mu_{x}(t)=\mu_{x}(t)\left(b_{x}+\frac{1}{2} \gamma^{2} b_{x}^{2}\right) d t+\gamma \mu_{x}(t) b_{x} d X_{t},
$$

which is a lognormal model for $\mu_{x}(t)$. In Pintoux and Privault [2011], a formula for $E\left(\exp \left(-\int_{t}^{T} \mu_{x}(s) d s\right)\right)$ under the lognormal model is supplied. However, we are interested in the mortality rate $\mu_{x+s}(s)$ in which the age subscript $x+s$ increases with time $s$. Employing the "affine matching principle" described in Duffie and Kan [1996], we can deduce a formula for $E\left(\exp \left(-\int_{t}^{T} \mu_{x+s}(s) d s\right)\right)$. The "affine matching principle" involves solving the Feynman-Kac partial differential equation associated to an affine stochastic process $Y$ by trialling a solution of the form $\exp \left\{\gamma(x, t, T)-Y_{t} \delta(x, t, T)\right\}$ and subsequently solving a system of ordinary differential equations in $\gamma$ and $\delta$. In Schrager [2006], this principle is applied to modelling the mortality intensity as a linear combination of affine stochastic processes, allowing tractability in the survivor functions. Maintaining tractability in our modelling, we model the primitive mortality rate $M$ in the current paper as a random process, employing a single factor Gaussian affine model with Gompertz-Makeham-Siler expected mortality growth rate satisfying the random walk SDE

$$
d M_{x+t}(t)=\left(a_{2} \exp \left(a_{0}+a_{1} x+a_{2} t\right)+b_{2} \exp \left(b_{0}+b_{1} x+b_{2} t\right)\right) d t+\beta d X_{t},
$$

or with mean reverting growth rate, as given by the extended Ornstein-Uhlenbeck SDE,

$d M_{x+t}(t)=\left(a_{2} \exp \left(a_{0}+a_{1} x+a_{2} t\right)+b_{2} \exp \left(b_{0}+b_{1} x+b_{2} t\right)-\gamma M_{x+t}(t)\right) d t+\beta d X_{t}$.

Here $X$ is a Wiener process, as in (3.17), adapted to the filtration $\mathcal{A}$ and which is independent of $Z$ and $W$, and $a_{0}, a_{1}, a_{2}, b_{0}, b_{1}, b_{2}, \beta$ and $\gamma$ are constants. We note that the first model in (3.19) is a particular case of the extended OrnsteinUhlenbeck model in (3.20) with $\gamma=0$. In Dahl and Moller [2006] a similar model is suggested.

3.4. Data and Parameter Estimation. Using standard maximum likelihood estimation, each of the considered models is fitted to market data composed of US oneyear cash rates and S\&P Composite Index values, obtained from Shiller [1989], and US life tables for males 1933-2015, sourced from the data set specified by subheadings USA, Period Data, Life tables, Males and Age interval x year interval $1 \mathrm{x} 1$ in the human mortality database (see http://www.mortality.org/hmd/USA/STATS/mltper_1x1.txt), with the additional simplifying assumption that no life survives to age 110 . 
Because the mortality models are Gaussian, maximum likelihood estimation of their parameters involves the normal probability density function, whose mean and variance are given in the following theorems. We abbreviate the expectation $E\left(\cdot \mid \mathcal{A}_{t}\right)$ and variance $\operatorname{VAR}\left(\cdot \mid \mathcal{A}_{t}\right)$ conditional on information up to time $t$ as $E_{t}(\cdot)$ and $V A R_{t}(\cdot)$, respectively.

Theorem 1. For the random walk model in (3.19) we have the statistics, for $s>t$,

$$
\begin{aligned}
& E_{t}\left(M_{x+s}(s)\right) \\
& =M_{x+t}(t)+\exp \left(a_{0}+a_{1} x+a_{2} t\right)\left\{\exp \left(a_{2}(s-t)\right)-1\right\} \\
& +\exp \left(b_{0}+b_{1} x+b_{2} t\right)\left\{\exp \left(b_{2}(s-t)\right)-1\right\} \\
& V A R_{t}\left(M_{x+s}(s)\right)=\beta^{2}(s-t) .
\end{aligned}
$$

Proof. Integrating both sides of (3.19) between times $t$ and $s$ gives

$$
M_{x+s}(s)=M_{x+t}(t)+\int_{t}^{s}\left\{a_{2} \exp \left(a_{0}+a_{1} x+a_{2} u\right)+b_{2} \exp \left(b_{0}+b_{1} x+b_{2} u\right)\right\} d u+\beta \int_{t}^{s} d X_{u},
$$

from which the result follows immediately.

Theorem 2. For the extended Ornstein-Uhlenbeck model in (3.20) we have the sufficient statistics, for $s>t$,

$$
\begin{aligned}
& E_{t}\left(M_{x+s}(s)\right)=M_{x+t}(t) \exp (-\gamma(s-t)) \\
& +\frac{a_{2}}{a_{2}+\gamma} \exp \left(a_{0}+a_{1} x+a_{2} t\right)\left\{\exp \left(a_{2}(s-t)\right)-\exp (-\gamma(s-t))\right\} \\
& +\frac{b_{2}}{b_{2}+\gamma} \exp \left(b_{0}+b_{1} x+b_{2} t\right)\left\{\exp \left(b_{2}(s-t)\right)-\exp (-\gamma(s-t))\right\} \\
& V A R_{t}\left(M_{x+s}(s)\right)=\beta^{2}(1-\exp \{-2 \gamma(s-t)\}) /(2 \gamma) .
\end{aligned}
$$

Proof. Writing $\nu_{t}=\exp (\gamma t) M_{x+t}(t)$ we have, from (3.20) and Ito's Lemma,

$$
\begin{aligned}
& d \nu_{t}=\exp (\gamma t) d M_{x+t}(t)+\gamma \exp (\gamma t) M_{x+t}(t) d t \\
& =\exp (\gamma t)\left(a_{2} \exp \left(a_{0}+a_{1} x+a_{2} t\right)+b_{2} \exp \left(b_{0}+b_{1} x+b_{2} t\right)\right) d t+\beta \exp (\gamma t) d X_{t} .
\end{aligned}
$$

Integrating both sides of the equation for $d \nu_{t}$ between times $t$ and $s$ gives

$$
\begin{aligned}
\nu_{s} & =\nu_{t}+\frac{a_{2}}{a_{2}+\gamma} \exp \left(a_{0}+a_{1} x+a_{2} t\right)\left\{\exp \left(a_{2}(s-t)\right) \exp (\gamma s)-\exp (\gamma t)\right\} \\
& +\frac{b_{2}}{b_{2}+\gamma} \exp \left(b_{0}+b_{1} x+b_{2} t\right)\left\{\exp \left(b_{2}(s-t)\right) \exp (\gamma s)-\exp (\gamma t)\right\} \\
& +\int_{t}^{s} \beta \exp (\gamma u) d X_{u} .
\end{aligned}
$$


TABle 2. Parameter values of discounted GOP models fitted to monthly series of discounted US S\&P Composite Index 1871-2018. Also shown are the standard errors in brackets, the log-likelihood $\ell$, the Akaike information criterion $A I C$ and Bayesian information criterion $B I C$.

\begin{tabular}{|c|c|}
\hline Black-Scholes & MMM \\
\hline$\theta=0.140592(0.002363)$ & $\bar{\alpha}_{0}=0.006910(0.000475)$ \\
\hline & $\eta=0.045290(0.000$ \\
\hline$A I C$ & $A I C=19$ \\
\hline$B I C=2237.61$ & $B I C=2007.56$ \\
\hline
\end{tabular}

Dividing both sides of the equation for $\nu_{s}$ by $\exp (\gamma s)$ gives

$$
\begin{aligned}
M_{x+s}(s) & =M_{x+t}(t) \exp (-\gamma(s-t)) \\
& +\frac{a_{2}}{a_{2}+\gamma} \exp \left(a_{0}+a_{1} x+a_{2} t\right)\left\{\exp \left(a_{2}(s-t)\right)-\exp (-\gamma(s-t))\right\} \\
& +\frac{b_{2}}{b_{2}+\gamma} \exp \left(b_{0}+b_{1} x+b_{2} t\right)\left\{\exp \left(b_{2}(s-t)\right)-\exp (-\gamma(s-t))\right\} \\
& +\int_{t}^{s} \beta \exp (-\gamma(s-u)) d X_{u},
\end{aligned}
$$

from which the result follows.

The obtained parameter values, together with log-likelihood values and standard errors, are shown in Tables 2, 3 and 5, under the assumption of independence, and, under the general model with $f^{r}(x)=f^{\mu}(x)=g^{\mu}(x)=x$, in Tables 4 and 6 , with $\alpha^{r} \approx \alpha^{\mu} \approx \beta^{\mu} \approx 0$ suggesting little dependence.

The fitting of the short rate models is described in Fergusson and Platen [2015b] and the fitting of the discounted stock index models is described in Fergusson [2017b]. The fitting of the mortality models is achieved by maximising the loglikelihood function

$$
\ell\left(a_{0}, a_{1}, a_{2}, \ldots, \beta\right)=\sum_{x=0}^{109} \sum_{t=1933}^{2014} \log f_{\mu_{x+t+1}(t+1) \mid \mathcal{A}_{t}}\left(\hat{\mu}_{x+t+1}(t+1)\right)
$$

with the Newton-Raphson iterative method, using initial estimates of the nascent and senescent parameters derived by regressing the young age mortality rates (ages $0-18$ ) and older age mortality rates (ages 18-110) on corresponding values of $x$ and $t$.

The suitability of these processes for describing the real world has been supported by statistical analyses of Chan et al. [1992], Aït-Sahalia [1996] in respect of short rates, Fergusson [2017a] in respect of discounted indices, and Schrager [2006] in respect of mortality. For the $3 / 2$ model, simulations using the fitted parameters in Table 3 give rise to large, yet short-lived, values. However, it is included here to highlight the variety of short rate models accommodated in the general framework and to accomodate the documented $r_{t}^{3 / 2}$ behaviour in the short rate diffusion. 
TABLE 3. Parameter values of short rate models fitted to monthly series of US one-year cash rates 1871-2018. Also shown are the standard errors in brackets, the log-likelihood $\ell$, the Akaike information criterion $A I C$ and Bayesian information criterion $B I C$.

\begin{tabular}{|c|c|c|}
\hline Vasicek & CIR & $3 / 2$ \\
\hline $\bar{r}=0.043591(0.008539)$ & $\bar{r}=0.042567(0.011230)$ & $p=0.109747(0.038977)$ \\
\hline$\kappa=0.149683(0.045449)$ & $\kappa=0.094675(0.034991)$ & $q=-0.009232(1.219036)$ \\
\hline $\begin{array}{l}\sigma=0.015479(0.000262) \\
---\ell^{-}=7064.4 \overline{9}---\end{array}$ & $\begin{array}{c}\sigma=0.064411(0.001089) \\
---\bar{\ell}=\overline{7} 51 \overline{10.57^{-}--}\end{array}$ & $\begin{array}{c}\sigma=2.256888(0.038186) \\
---\bar{\ell}=\overline{7} 1 \overline{6} \overline{4} . \overline{35}---\end{array}$ \\
\hline$A I C=-14122.98$ & $A I C=-15015.14$ & $A I C=-14322.70$ \\
\hline$B I C=-14106.55$ & $B I C=-14998.70$ & $B I C=-14306.27$ \\
\hline
\end{tabular}

TABle 4. Parameter values of short rate models with $f^{r}(x)=x$ fitted to monthly series of US one-year cash rates 1871-2018. Also shown are the standard errors in brackets, the log-likelihood $\ell$, the Akaike information criterion $A I C$ and Bayesian information criterion $B I C$.

\begin{tabular}{ccc}
\hline Vasicek & CIR & $3 / 2$ \\
\hline $\bar{r}=0.046275(0.008727)$ & $\bar{r}=0.045268(0.015002)$ & $p=0.095320(0.030828)$ \\
$\kappa=0.147341(0.045759)$ & $\kappa=0.096647(0.044971)$ & $q=-0.508337(0.966814)$ \\
$\sigma=0.015474(0.000262)$ & $\sigma=0.062509(0.001011)$ & $\sigma=1.785932(0.021922)$ \\
$\alpha^{r}=-0.000976(0.000995)$ & $\alpha^{r}=-0.000976(0.000445)$ & $\alpha^{r}=-0.000976(0.000102)$ \\
$-\bar{\ell}=7064.9 \overline{1}$ & AIC $=-14949.43$ & $A I C=-14636.65$ \\
$A I C=-14121.82$ & $B I C=-14927.53$ & $B I C=-14614.74$ \\
$B I C=-14099.92$ &
\end{tabular}

TABLE 5. Parameter values of mortality models fitted to US life tables of males 1933-2015. Also shown are the standard errors in brackets, the log-likelihood $\ell$, the Akaike information criterion $A I C$ and Bayesian information criterion BIC.

\begin{tabular}{cc}
\hline Gompertz-Makeham-Siler RW & Gompertz-Makeham-Siler OU \\
\hline$a_{0}=-8.111622(0.004669)$ & $a_{0}=-6.050464(0.003458)$ \\
$a_{1}=0.067995(0.000068)$ & $a_{1}=0.052635(0.000053)$ \\
$a_{2}=0.068574(0.000076)$ & $a_{2}=0.053671(0.000052)$ \\
$b_{0}=-11.917990(0.000000)$ & $b_{0}=-2.906129(0.065398)$ \\
$b_{1}=-0.097077(0.000000)$ & $b_{1}=-0.858624(0.106140)$ \\
$b_{2}=-0.436887(0.000000)$ & $b_{2}=-17.334363(0.000000)$ \\
& $\gamma=0.013902(0.000218)$ \\
$\beta=0.008119(0.000030)$ & $\beta=0.007818(0.000029)$ \\
\hline$\ell=\overline{3} 0 \overline{6} \overline{4} \overline{4} . \overline{3} \overline{8}$ & $\bar{\ell}=\overline{3} \overline{1} 0 \overline{2} \overline{2} . \overline{8} 6^{-}---$ \\
$A I C=-61274.76$ & $A I C=-62029.71$ \\
$B I C=-61225.01$ & $B I C=-61972.86$ \\
\hline
\end{tabular}


TABle 6. Parameter values of mortality models with $f^{r}(x)=x$, $f^{\mu}(x)=x$ and $g^{\mu}(x)=x$, fitted to US life tables of males 19332015. Also shown are the standard errors in brackets, the loglikelihood $\ell$, the Akaike information criterion $A I C$ and Bayesian information criterion $B I C$.

\begin{tabular}{|c|c|}
\hline Gompertz-Makeham-Siler RW & Gompertz-Makeham-Siler OU \\
\hline$a_{0}=-8.089649(0.004644)$ & $a_{0}=-6.043137(0.003471)$ \\
\hline$a_{1}=0.067251(0.000069)$ & $a_{1}=0.052535(0.000053)$ \\
\hline$a_{2}=0.068941(0.000074)$ & $a_{2}=0.053586(0.000052)$ \\
\hline$b_{0}=-11.632368(1.406302)$ & $b_{0}=-2.891557(0.012378)$ \\
\hline$b_{1}=-0.074116(0.000000)$ & $b_{1}=-0.823818(0.094591)$ \\
\hline$b_{2}=-0.310243(0.000000)$ & $b_{2}=-17.372233(0.000000)$ \\
\hline & $\gamma=0.014008(0.000218)$ \\
\hline$\beta=0.008059(0.000030)$ & $\beta=0.007796(0.000029)$ \\
\hline$\alpha^{\mu}=0.002063(0.000086)$ & $\alpha^{\mu}=0.002063(0.000014)$ \\
\hline$\beta^{\mu}=-0.016400(0.000033)$ & $\beta^{\mu}=-0.016400(0.000014)$ \\
\hline & $\bar{\ell}=\overline{3} \overline{1} 0 \overline{2} . \overline{17}$ \\
\hline$A I C=-61383.30$ & $A I C=-62030.34$ \\
\hline$B I C=-61319.34$ & $B I C=-61973.48$ \\
\hline
\end{tabular}

\section{Determining the Valuation Formulae}

Prior to giving the valuation formulae for the variable annuity and its components described in Section 2, we first clarify the notation and provide some propositions which are used in deriving the valuation formulae. Then, in the following three subsections, namely Subsections 4.1, 4.2 and 4.3, valuation formulae for our previously-mentioned variable annuity and its component benefits are determined.

Let us denote by $\tau_{x+t}(t)$ the time of death of a life aged $x+t$ at time $t$. In respect of a life aged $x$, the standard actuarial notations for the one-year survival probability, the $n$-year survival probability, the one-year death probability and the $n$-year death probability are $p_{x},{ }_{n} p_{x}, q_{x}$ and ${ }_{n} q_{x}$, respectively. Typically these probabilities are tabulated in a static life table. In keeping with this notation, and allowing for dynamic life tables, we employ the corresponding time-dependent notation $p_{x}(t),{ }_{n} p_{x}(t), q_{x}(t)$ and ${ }_{n} q_{x}(t)$, in respect of a life aged $x$ at time $t$. Further, when our probabilities are estimated using a mortality model, we use the corresponding notations $\hat{p}_{x}(t),{ }_{n} \hat{p}_{x}(t), \hat{q}_{x}(t)$ and ${ }_{n} \hat{q}_{x}(t)$.

Also, let us denote by $\tau_{x+t}^{(D)}(t)$ the time of death of a policyholder, who has not previously lapsed, aged $x+t$ at time $t$, so that for $t \in\left[t_{j}, t_{j+1}\right)$ and $i \in\{j+1, \ldots, n\}$, $P\left(\tau_{x+t}^{(D)}(t)>t_{i}\right)=P\left(\tau_{x+t}(t)>t_{i}\right)(1-\rho)^{i-j}$.

Lastly, we define the expectation, in respect of a stochastic process $U=\left\{U_{t}\right.$ : $t \geq 0\}$, with $U_{0}=x$,

$\mathcal{E}(t, x, U, \alpha, \mu, \nu, \xi)=E\left(U_{t}^{-\alpha} \exp \left\{-\mu \int_{0}^{t} U_{s} d s-\nu \int_{0}^{t} \frac{1}{U_{s}} d s-\xi \int_{0}^{t} \log \left(U_{s}\right) d s\right\}\right)$.

We now state three propositions which give closed-form formulae for this expectation for relevant stochastic processes, where the proofs of the first two are straightforward and the third proof is given in Craddock and Lennox [2009]. 
Proposition 1. Assume that $U$ is given by $d U_{t}=a U_{t} d t+\sigma U_{t} d W_{t}$, where $U_{0}=x$. Define $m=(\alpha+\xi t) \log x+\left(\alpha t+\frac{1}{2} \xi t^{2}\right)\left(a-\frac{1}{2} \sigma^{2}\right)$ and $k=\sigma^{2} t\left(\alpha^{2}+\alpha \xi t+\frac{1}{3} \xi^{2} t^{2}\right)$. Then

$$
\mathcal{E}(t, x, U, \alpha, 0,0, \xi)=\exp \left\{-m+\frac{1}{2} k\right\} .
$$

Proposition 2. Assume that $U$ is given by $d U_{t}=\left(a-b U_{t}\right) d t+\sigma d W_{t}$, where $U_{0}=$ $x$. Define $\beta=\{1-\exp (-b t)\} / b, m=x \beta+a t / b-a \beta / b$ and $k=\sigma^{2}(t-\beta+b \beta / 2) / b^{2}$. Then

$$
\mathcal{E}(t, x, U, 0, \mu, 0,0) \equiv E\left(\exp \left\{-\mu \int_{0}^{t} U_{s} d s\right\}\right)=\exp \left\{-m+\frac{1}{2} k\right\} .
$$

Finally, we make use of Proposition 7.3.9 presented in Baldeaux and Platen [2013], concerning benchmarked Laplace transforms, and restated as follows.

Proposition 3. Assume that $U=\left\{U_{t}: t \geq 0\right\}$ is given by $d U_{t}=\left(a-b U_{t}\right) d t+$ $\sqrt{\sigma U_{t}} d W_{t}$, where $U_{0}=x$, and that $2 a / \sigma \geq 2$.

Define $A=b^{2}+4 \mu \sigma, m=\frac{1}{\sigma} \sqrt{(a-\sigma)^{2}+4 \sigma \nu}, \beta=\sqrt{A x} /\{\sigma \sinh (\sqrt{A} t / 2)\}$ and $k=\{\sqrt{A}+b \tanh (\sqrt{A} t / 2)\} /\{2 \sigma \tanh (\sqrt{A} t / 2)\}$.

Then if $a>(2 \alpha-3) \sigma$, for $\mu>0, \nu \geq 0$,

$$
\begin{aligned}
& E\left(U_{t}^{-\alpha} \exp \left\{-\nu \int_{0}^{t} \frac{d s}{U_{s}}-\mu \int_{0}^{t} U_{s} d s\right\}\right)=\frac{\sqrt{A} x^{1 / 2-a /(2 \sigma)}}{2 \sigma \sinh (\sqrt{A} t / 2)}(\beta / 2)^{m} \\
& \quad \times \exp \left\{\frac{b(x+a t)-\sqrt{A} x \operatorname{coth}(\sqrt{A} t / 2)}{2 \sigma}\right\} k^{-\left(1-\alpha+\frac{a}{2 \sigma}+\frac{1}{2}+\frac{m}{2}\right)} \\
& \quad \times \frac{\Gamma\left(1-\alpha+\frac{a}{2 \sigma}+\frac{1}{2}+\frac{m}{2}\right)}{\Gamma(1+m)}{ }_{1} F_{1}\left(1-\alpha+\frac{a}{2 \sigma}+\frac{1}{2}+\frac{m}{2}, 1+m, \frac{\beta^{2}}{4 k}\right) .
\end{aligned}
$$

4.1. Valuation Formulae for the Surrender Benefit. Under our considered market models, the real-world valuation formula (A.1) applied to the valuation of a contingent claim having payoff given in (2.3) at time $t_{i}$ involves the evaluation of the expectation

$$
V_{t, t_{i}}^{(L)}=1_{\tau_{x+t}^{(L)}(t)>t} E_{t}\left(\frac{S_{t}}{S_{t_{i}}} H_{t_{i}}^{(L)} 1_{\tau_{x+t}^{(L)}(t) \in\left[t_{i-1}, t_{i}\right)}\right),
$$

which can be rewritten as

$$
\begin{aligned}
V_{t, t_{i}}^{(L)} & =E_{t}\left(\exp \left\{-\int_{t}^{t_{i}} \mu_{x+s}(s) d s-\int_{t}^{t_{i}} r_{s} d s\right\} \frac{\bar{\alpha}_{t} Y_{t}}{\bar{\alpha}_{t_{i}} Y_{t_{i}}}\right) \sqrt{K_{t_{i}}}(1-\rho)^{i-1-j} \rho \\
& =P_{x+t}\left(t, t_{i}\right) \sqrt{K_{t_{i}}}(1-\rho)^{i-1-j} \rho,
\end{aligned}
$$

where $P_{x+t}(t, T)$ denotes the time- $t$ value of a $T$-maturity survivor ZCB in respect of a life aged $x+t$ at time $t$, where a payment of $\$ 1$ is paid if the life survives to maturity.

Theorem 3. For $t \in\left[t_{j}, t_{j+1}\right)$, the real-world value of the surrender benefit paid at time $t_{i}$ is

$$
V_{t, t_{i}}^{(L)}=V_{t, t_{i}}(Y) \times V_{t, t_{i}}(R) \times V_{t, t_{i}, x}(M) \times \sqrt{K_{t_{i}}}(1-\rho)^{i-j-1} \rho,
$$


where

$$
\begin{aligned}
V_{t, t_{i}}(Y) & =E_{t}\left(\exp \left\{-\int_{t}^{t_{i}} \alpha^{r} f^{r}\left(Y_{s}\right)+\alpha^{\mu} f^{\mu}\left(Y_{s}\right) d s\right\} \frac{\bar{\alpha}_{t} Y_{t}}{\bar{\alpha}_{t_{i}} Y_{t_{i}}}\right) \equiv \frac{\bar{\alpha}_{t} Y_{t}}{\bar{\alpha}_{t_{i}}} \mathcal{E}\left(t_{i}-t, Y_{t}, Y\right) \\
V_{t, t_{i}}(R) & =E_{t}\left(\exp \left\{-\int_{t}^{t_{i}}\left(\beta^{\mu} g^{\mu}\left(R_{s}\right)+R_{s}\right) d s\right\}\right)=\mathcal{E}\left(t_{i}-t, R_{t}, R\right) \\
V_{t, t_{i}, x}(M) & =E_{t}\left(\exp \left\{-\int_{t}^{t_{i}} M_{x+s}(s) d s\right\}\right),
\end{aligned}
$$

where the omitted final four arguments in $\mathcal{E}$ are to be understood from the context.

Proof. We can decompose $V_{t, t_{i}}^{(L)}$ into a product of independent stochastic exponentials

$$
V_{t, t_{i}}^{(L)}=V_{t, t_{i}}(Y) \times V_{t, t_{i}}(R) \times V_{t, t_{i}, x}(M) \times(1-\rho)^{i-j-1} \rho,
$$

where $V_{t, t_{i}}(Y), V_{t, t_{i}}(R)$ and $V_{t, t_{i}, x}(M)$ are as in (4.8).

We call $V_{t, t_{i}}(Y), V_{t, t_{i}}(R)$ and $V_{t, t_{i}, x}(M)$ the contributions to the survivor ZCB from the discounted GOP, primitive short rate and primitive mortality rate respectively.

As an immediate corollary, when $\alpha^{r}=\alpha^{\mu}=\beta^{\mu}=0$, under our considered market models, where the driving Brownian motions are assumed to be independent, the real-world valuation formula (A.1) gives the value of a survivor ZCB as

$$
P_{x+t}(t, T)=E_{t}\left(\exp \left(-\int_{t}^{T} \mu_{x+s}(s) d s\right)\right) E_{t}\left(\frac{B_{t}}{B_{T}}\right) E_{t}\left(\frac{\bar{S}_{t}}{\bar{S}_{T}}\right)={ }_{T-t} \hat{p}_{x+t}(t) P(t, T),
$$

where $P(t, T)$ is the time- $t$ value of a $T$-maturity ZCB.

The contribution to the survivor ZCB from the discounted GOP when $\bar{S}$ obeys the Black-Scholes model in (3.3) and $f^{r}(x)=f^{\mu}(x)=\log x$ can be deduced from Proposition 1, to be

$V_{t, t_{i}}(Y)=\exp \left\{-\left(t_{i}-t\right)\left(\alpha^{r}+\alpha^{\mu}\right)\left(\log x-\frac{1}{2}\left(t_{i}-t\right) \eta-\frac{1}{6} \theta^{2}\left(t_{i}-t\right)^{2}\left(\alpha^{r}+\alpha^{\mu}\right)\right)\right\}$,

simplifying to unity when $\alpha^{r}=\alpha^{\mu}=0$.

The contribution to the survivor ZCB from the discounted GOP, when $\bar{S}$ obeys the MMM in (3.4) and each of the functions $f^{r}(x)$ and $f^{\mu}(x)$ is either of $x$ or $1 / x$, can be obtained using Proposition 3. In the particular case $\alpha^{r}=\alpha^{\mu}=0$ we obtain, in agreement with Platen and Heath [2006],

$$
V_{t, t_{i}}(Y)=E_{t}\left(\frac{\bar{S}_{t}}{\bar{S}_{t_{i}}}\right)=1-\exp \left(-\frac{1}{2} \bar{S}_{t} /\left(\varphi_{t_{i}}-\varphi_{t}\right)\right)
$$

where $\varphi_{t}=\frac{1}{4} \bar{\alpha}_{0}(\exp (\eta t)-1) / \eta$. In this case $\left(\bar{S}_{t}\right)^{-1}$ is a strict local martingale that reflects much more realistically the long-term dynamics of the benchmarked savings account than given by a Black-Scholes model.

The contributions from the from the primitive short rate $R$ when $\beta^{\mu}=0$ for the Vasicek, CIR and 3/2 models are given in Vasicek [1977], Cox et al. [1985] and Ahn and Gao [1999], respectively. For $\beta^{\mu} \neq 0$, when $R$ is either a CIR or $3 / 2$ process and $g^{\mu}(x)$ is either $x$ or $1 / x$, the contribution to the survivor ZCB follows 
directly from Proposition 3, and when $R$ obeys the Vasicek model and $g^{\mu}(x)=x$, the contribution is deduced from Proposition 2.

We now give formulae for the contributions $V_{t, t_{i}, x}(M)$ to the survivor ZCB from the primitive mortality rate under each of the mortality models in the following two theorems. By way of notation, we write for the survival probability to time $T$, in respect of a life aged $x+t$ at time $t$,

$$
{ }_{T-t} \hat{p}_{x+t}(t) \equiv E_{t}\left(\exp \left\{-\int_{t}^{T} \mu_{x+s}(s) d s\right\}\right)
$$

and for the survival probability due to the primitive mortality rate only,

$$
{ }_{T-t} \hat{p}_{x+t}^{(M)}(t) \equiv V_{t, T, x}(M) \equiv E_{t}\left(\exp \left\{-\int_{t}^{T} M_{x+s}(s) d s\right\}\right)
$$

where agreement between the two is obtained when $\alpha^{\mu}=\beta^{\mu}=0$.

Theorem 4. For the random walk model in (3.19) we have the survival probability

$$
\begin{aligned}
& T-t \hat{p}_{x+t}^{(M)}(t)=E_{t}\left(\exp \left(-\int_{t}^{T} M_{x+s}(s) d s\right)\right) \\
& =\exp \left\{-\left\{M_{x+t}(t)-\exp \left(a_{0}+a_{1} x+a_{2} t\right)-\exp \left(b_{0}+b_{1} x+b_{2} t\right)\right\}(T-t)\right. \\
& -\frac{1}{a_{2}} \exp \left(a_{0}+a_{1} x+a_{2} t\right)\left(\exp \left\{a_{2}(T-t)\right\}-1\right) \\
& \left.-\frac{1}{b_{2}} \exp \left(b_{0}+b_{1} x+b_{2} t\right)\left(\exp \left\{b_{2}(T-t)\right\}-1\right)+\frac{1}{6} \beta^{2}(T-t)^{3}\right\}
\end{aligned}
$$

Proof. Integrating both sides of (3.22) we see that $\int_{t}^{T} M_{x+s}(s) d s$ is a normally distributed random variable having mean and variance

$$
\begin{aligned}
& E_{t}\left(\int_{t}^{T} M_{x+s}(s) d s\right)=\left\{M_{x+t}(t)-\exp \left(a_{0}+a_{1} x+a_{2} t\right)-\exp \left(b_{0}+b_{1} x+b_{2} t\right)\right\}(T-t) \\
& +\frac{1}{a_{2}} \exp \left(a_{0}+a_{1} x+a_{2} t\right)\left(\exp \left\{a_{2}(T-t)\right\}-1\right) \\
& +\frac{1}{b_{2}} \exp \left(b_{0}+b_{1} x+b_{2} t\right)\left(\exp \left\{b_{2}(T-t)\right\}-1\right), \\
& V A R_{t}\left(\int_{t}^{T} M_{x+s}(s) d s\right)=V A R_{t}\left(\int_{t}^{T}\left(\int_{t}^{s} \beta d X_{u}\right) d s\right) \\
& =V A R_{t}\left(\beta \int_{t}^{T}\left(\int_{u}^{T} d s\right) d X_{u}\right)=V A R_{t}\left(\beta \int_{t}^{T}(T-u) d X_{u}\right) \\
& =\beta^{2} \int_{t}^{T}(T-u)^{2} d u=\frac{1}{3} \beta^{2}(T-t)^{3} .
\end{aligned}
$$

The result follows straightforwardly from the formula for the mean of a lognormal random variable. 
Theorem 5. For the extended Ornstein-Uhlenbeck model in (3.20) we have the survival probability

$$
\begin{aligned}
& \left.T-t \hat{p}_{x+t}^{(M)}(t) \equiv E_{t}\left(\exp \left(-\int_{t}^{T} M_{x+s}(s) d s\right)\right)\right) \\
& =\exp \left(-M_{x+t}(t) B(t, T)-\frac{a_{2}}{a_{2}+\gamma}\left\{\frac{1}{a_{2}}\left(\exp \left(a_{2}(T-t)\right)-1\right)-B(t, T)\right\}\right. \\
& \left.-\frac{b_{2}}{b_{2}+\gamma}\left\{\frac{1}{b_{2}}\left(\exp \left(b_{2}(T-t)\right)-1\right)-B(t, T)\right\}+\frac{1}{2} \beta^{2} \frac{1}{\gamma^{2}}\left(T-t-B(t, T)-\frac{\gamma}{2} B(t, T)^{2}\right)\right),
\end{aligned}
$$

where

$$
B(t, T)=\frac{1}{\gamma}(1-\exp (-\gamma(T-t))) .
$$

Proof. Integrating both sides of (3.26) from $s=t$ to $s=T$ we see that the integral $\int_{t}^{T} M_{x+s}(s) d s$ is normally distributed with mean and variance

$$
\begin{aligned}
& E_{t}\left(\int_{t}^{T} M_{x+s}(s) d s\right)=M_{x+t}(t) \frac{1}{\gamma}(1-\exp (-\gamma(T-t))) \\
&+\frac{a_{2}}{a_{2}+\gamma}\left\{\frac{1}{a_{2}}\left(\exp \left(a_{2}(T-t)\right)-1\right)-B(t, T)\right\} \\
&+\frac{b_{2}}{b_{2}+\gamma}\left\{\frac{1}{b_{2}}\left(\exp \left(b_{2}(T-t)\right)-1\right)-B(t, T)\right\}, \\
& V A R_{t}\left(\int_{t}^{T} M_{x+s}(s) d s\right)=\beta^{2} \int_{t}^{T} B(u, T)^{2} d u \\
&=\beta^{2} \frac{1}{\gamma^{2}} \int_{t}^{T} 1-2 \exp (-\gamma(T-u))+\exp (-2 \gamma(T-u)) d u \\
&=\beta^{2} \frac{1}{\gamma^{2}}\left(T-t-2 \frac{1}{\gamma}(1-\exp (-\gamma(T-t)))+\frac{1}{2 \gamma}(1-\exp (-2 \gamma(T-t)))\right) \\
&=\beta^{2} \frac{1}{\gamma^{2}}\left(T-t-B(t, T)-\frac{\gamma}{2} B(t, T)^{2}\right) .
\end{aligned}
$$

Using the formula for the mean of the lognormal distribution, the result follows.

The formulae for the expectation of the exponential integrals of mortality rates correspond to the survival probability functions whose graphs are shown in Figures 1 and 2 for the parameter values in Table 5. The graphed survival probability functions exhibit the stylised property of being monotone decreasing and finally asymptotically approaching zero. Because we are using at most eight parameters in our mortality models, the fitted survival probabilities are by no means as precise as would be the case using a Lee-Carter model. However, this illustrates the trend risk, the systematic shift in mortality rates, to which insurers are exposed. Also, the imprecise fitting of survival probabilities highlights the problem faced by insurers, whereby money invested in actuarial assets needs to accommodate deviations of mortality experienced from that which is modelled and, therefore, the actuarial assets may be different from those prescribed by the model. 
FiguRE 1. Graph of survival probability functions for a US male life aged 40 in 1933 and hypothetical lives under the random walk model and extended Ornstein-Uhlenbeck model.

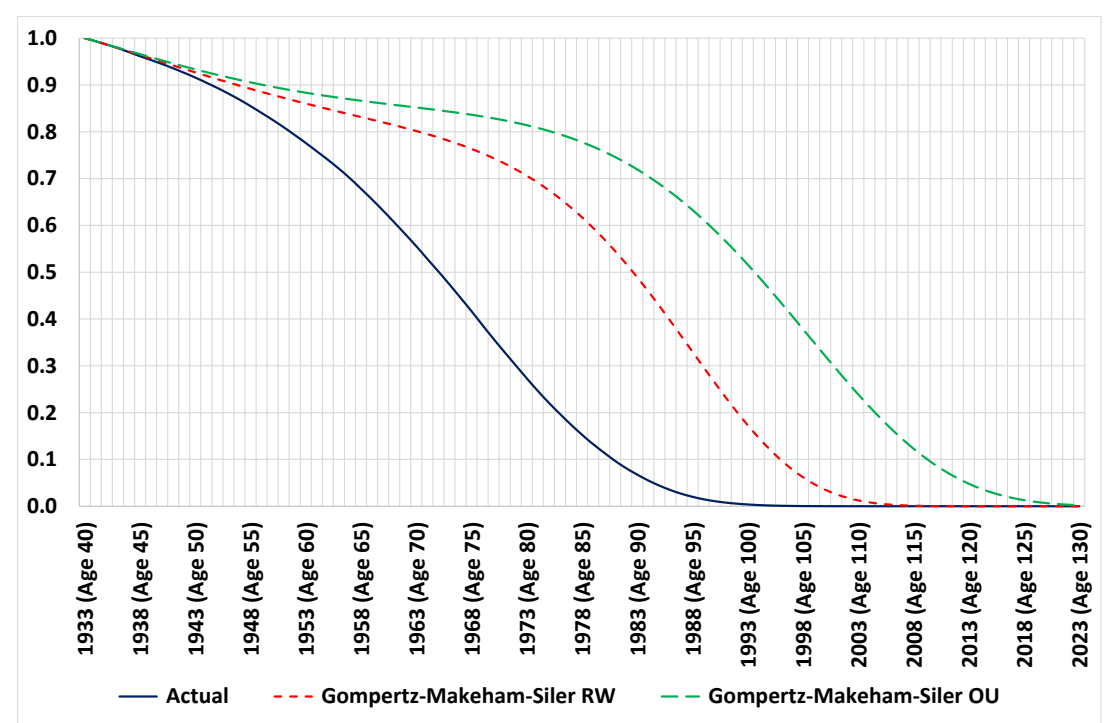

4.2. Valuation of Death Benefits and Accumulation Benefit. Application of the real-world valuation formula (A.1) to the payoff (2.1) at time $t_{i}$ involves the evaluation of the expectation

$$
V_{t, t_{i}}^{(D)}=1_{\tau_{x+t}^{(D)}(t)>t} E_{t}\left(\frac{S_{t}}{S_{t_{i}}} H_{t_{i}}^{(D)} 1_{\tau_{x+t}^{(D)}(t) \in\left[t_{i-1}, t_{i}\right)}\right) .
$$

We decompose the payoff in (2.1) into two parts, $H_{t_{i}}^{(D, 1)}$ and $H_{t_{i}}^{(D, 2)}$, given by

$$
H_{t_{i}}^{(D, 1)}=\frac{S_{t_{i}}}{S_{t_{0}}} \exp \left\{-\xi\left(t_{i}-t_{0}\right)\right\} \equiv F_{t_{i}}
$$

and

$$
H_{t_{i}}^{(D, 2)}=\left(K_{t_{i}}-\frac{S_{t_{i}}}{S_{t_{0}}} \exp \left\{-\xi\left(t_{i}-t_{0}\right)\right\}\right)^{+} \equiv\left(K_{t_{i}}-F_{t_{i}}\right)^{+}
$$

and we denote by $V_{t, t_{i}}^{(D, 1)}$ and $V_{t, t_{i}}^{(D, 2)}$ the corresponding values, so that $V_{t, t_{i}}^{(D)}=$ $V_{t, t_{i}}^{(D, 1)}+V_{t, t_{i}}^{(D, 2)}$. Also, application of the real-world valuation formula (A.1) to the payoff (2.4) at time $t_{n}$ involves the evaluation of the expectation

$$
V_{t, t_{n}}^{(A)}=1_{\tau_{x+t}^{(D)}(t)>t} E_{t}\left(\frac{S_{t}}{S_{t_{n}}} H_{t_{n}}^{(A)} 1_{\tau_{x+t}^{(D)}(t) \in\left[t_{n}, \infty\right)}\right),
$$


FiguRE 2. Graph of survival probability functions for a US male life aged 65 in 1971 and hypothetical lives under the random walk model and extended Ornstein-Uhlenbeck model.

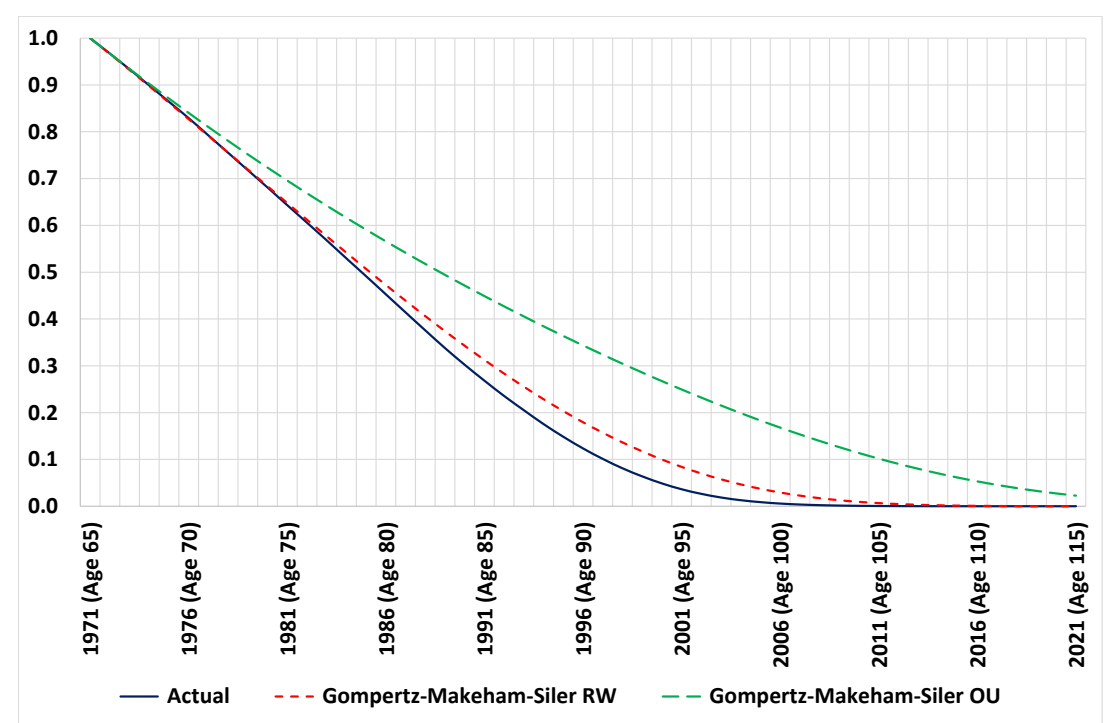

which can be rewritten as

$$
(1-\rho)^{n-j} E_{t}\left(\exp \left\{-\int_{t}^{t_{n}} \mu_{x+s}(s) d s-\int_{t}^{t_{n}} r_{s} d s\right\} \frac{\alpha_{t} Y_{t}}{\alpha_{t_{n}} Y_{t_{n}}} H_{t_{n}}^{(A)}\right) .
$$

As for the death payoff, we decompose the payoff in (2.4) into three parts, $H_{t_{n}}^{(A, 1)}$, $H_{t_{n}}^{(A, 2)}$ and $H_{t_{n}}^{(A, 3)}$, where $H_{t_{n}}^{(A, 1)}=H_{t_{n}}^{(D, 1)}, H_{t_{n}}^{(A, 2)}=H_{t_{n}}^{(D, 2)}$ and

$$
H_{t_{n}}^{(A, 3)}=\left\{\frac{S_{t_{n}}}{S_{t_{0}}} \exp \left\{-\xi\left(t_{i}-t_{0}\right)\right\}-\left(K_{t_{n}}\right)^{3}\right\}^{+} \equiv\left(F_{t_{n}}-\left(K_{t_{n}}\right)^{3}\right)^{+},
$$

and we denote by $V_{t, t_{n}}^{(A, 1)}, V_{t, t_{n}}^{(A, 2)}$ and $V_{t, t_{n}}^{(A, 3)}$ the corresponding values, so that $V_{t, t_{n}}^{(A)}=$ $V_{t, t_{n}}^{(A, 1)}+V_{t, t_{n}}^{(A, 2)}-V_{t, t_{n}}^{(A, 3)}$.

The following lemma from Filipović [2009] will be employed in determining the values $V_{t, t_{i}}^{(D, k)}, V_{t, t_{n}}^{(A, \ell)}$ for $k \in\{1,2\}$ and $\ell \in\{1,2,3\}$.

Lemma 1. Let $K>0$. For any $y \in \mathbb{R}$ the following identities hold:

$$
\begin{aligned}
& \frac{1}{2 \pi} \int_{\mathbb{R}} \exp \{(w+\iota \lambda) y\} \frac{K^{-(w-1+\iota \lambda)}}{(w+\iota \lambda)(w-1+\iota \lambda)} d \lambda \\
& = \begin{cases}\left(K-\mathrm{e}^{y}\right)^{+}, & \text {if } w<0 ; \\
\left(\mathrm{e}^{y}-K\right)^{+}-\mathrm{e}^{y}, & \text { if } 0<w<1 ; \\
\left(\mathrm{e}^{y}-K\right)^{+}, & \text {if } w>1 .\end{cases}
\end{aligned}
$$


Theorem 6. The real-world values of the components of the death and accumulation benefits are

$$
\begin{aligned}
& V_{t, t_{i}}^{(D, 1)}=(1-\rho)^{i-1-j} \frac{S_{t}}{S_{t_{0}}} \exp \left\{-\xi\left(t_{i}-t_{0}\right)\right\}\left({ }_{i-j-1} \hat{p}_{x+t_{j}}\left(t_{j}\right)-{ }_{i-j} \hat{p}_{x+t_{j}}\left(t_{j}\right)\right) \\
& V_{t, t_{i}}^{(D, 2)}=V_{t, t_{i}}^{(D, 2 a)}-V_{t, t_{i}}^{(D, 2 b)} \\
& V_{t, t_{i}}^{(D, 2 a)}=(1-\rho)^{i-1-j} K_{t_{i}} \frac{1}{2 \pi} \int_{\mathbb{R}} \mathcal{E}_{t, t_{i}}^{(D)} \frac{\tilde{K}_{t_{i}}^{-(w-1+\iota \lambda)}}{(w+\iota \lambda)(w-1+\iota \lambda)} d \lambda
\end{aligned}
$$

$$
V_{t, t_{i}}^{(D, 2 b)}=(1-\rho)^{i-1-j} K_{t_{i}} \frac{1}{2 \pi} \int_{\mathbb{R}} \mathcal{E}_{t, t_{i}}^{(A)} \frac{\tilde{K}_{t_{i}}^{-(w-1+\iota \lambda)}}{(w+\iota \lambda)(w-1+\iota \lambda)} d \lambda
$$

$$
\begin{aligned}
& V_{t, t_{n}}^{(A, 1)}=(1-\rho)^{n-j} \exp \left\{-\xi\left(t_{n}-t_{0}\right)\right\}_{n-j} \hat{p}_{x+t_{j}}\left(t_{j}\right) \\
& V_{t, t_{n}}^{(A, 2)}=(1-\rho)^{n-j} K_{t_{n}} \frac{1}{2 \pi} \int_{\mathbb{R}} \mathcal{E}_{t, t_{n}}^{(A)} \frac{\tilde{K}_{t_{n}}^{-(w-1+\iota \lambda)}}{(w+\iota \lambda)(w-1+\iota \lambda)} d \lambda \\
& V_{t, t_{n}}^{(A, 3)}=(1-\rho)^{n-j} K_{t_{n}} \frac{1}{2 \pi} \int_{\mathbb{R}} \mathcal{E}_{t, t_{n}}^{(A)} \frac{\left(\tilde{\tilde{K}}_{t_{n}}\right)^{-(w-1+\iota \lambda)}}{(w+\iota \lambda)(w-1+\iota \lambda)} d \lambda
\end{aligned}
$$

where $\mathcal{E}_{t, t_{i}}^{(D)}$ and $\mathcal{E}_{t, t_{i}}^{(A)}$ are given by

$$
\begin{aligned}
& \mathcal{E}_{t, t_{i}}^{(D)}=E_{t}\left[\exp \left\{-\int_{t}^{t_{i-1}} \mu_{x+s}(s) d s\right\} \exp \left\{(w+\iota \lambda) \log \left(S_{t} / S_{t_{i}}\right)\right\}\right] \\
& \mathcal{E}_{t, t_{i}}^{(A)}=E_{t}\left[\exp \left\{-\int_{t}^{t_{i}} \mu_{x+s}(s) d s\right\} \exp \left\{(w+\iota \lambda) \log \left(S_{t} / S_{t_{i}}\right)\right\}\right]
\end{aligned}
$$

and

$$
\tilde{K}_{t_{i}}=\frac{S_{t}}{K_{t_{i}} S_{t_{0}}} \exp \left\{-\xi\left(t_{i}-t_{0}\right)\right\}, \quad \tilde{\tilde{K}}_{t_{n}}=\frac{S_{t}}{\left(K_{t_{n}}\right)^{3} S_{t_{0}}} \exp \left\{-\xi\left(t_{n}-t_{0}\right)\right\}
$$

Proof. We apply the real-world valuation formula in (A.1) to each of the payoffs $H_{t_{i}}^{(D, \cdot)}, H_{t_{n}}^{(A, \cdot)}$. Noting that

$$
E_{t}\left(1_{\tau_{x+t}^{(D)}(t) \in\left[t_{i-1}, t_{i}\right)}\right)=(1-\rho)^{i-j-1} E_{t}\left(1_{\tau_{x+t}(t) \in\left[t_{i-1}, t_{i}\right)}\right),
$$

we have for the first death payoff,

$$
\begin{aligned}
& V_{t, t_{i}}^{(D, 1)}=(1-\rho)^{i-1-j} E_{t}\left(\frac { S _ { t } } { S _ { t _ { i } } } H _ { t _ { i } } ^ { ( D , 1 ) } \left(\exp \left\{-\int_{t}^{t_{i-1}} \mu_{x+s}(s) d s\right\}\right.\right. \\
& \left.\left.-\exp \left\{-\int_{t}^{t_{i}} \mu_{x+s}(s) d s\right\}\right)\right) \\
& =(1-\rho)^{i-1-j} \frac{S_{t}}{S_{t_{0}}} \exp \left\{-\xi\left(t_{i}-t_{0}\right)\right\}\left(t_{i-1}-t_{j} \hat{p}_{x+t_{j}}\left(t_{j}\right)-t_{i}-t_{j} \hat{p}_{x+t_{j}}\left(t_{j}\right)\right) .
\end{aligned}
$$


For the second death payoff,

$$
\begin{aligned}
V_{t, t_{i}}^{(D, 2)}= & (1-\rho)^{i-1-j} E_{t}\left(\exp \left\{-\int_{t}^{t_{i-1}} \mu_{x+s}(s) d s\right\} \frac{S_{t}}{S_{t_{i}}} H_{t_{i}}^{(D, 2)}\right) \\
& -(1-\rho)^{i-1-j} E_{t}\left(\exp \left\{-\int_{t}^{t_{i}} \mu_{x+s}(s) d s\right\} \frac{S_{t}}{S_{t_{i}}} H_{t_{i}}^{(D, 2)}\right) \equiv V_{t, t_{i}}^{(D, 2 a)}-V_{t, t_{i}}^{(D, 2 b)} .
\end{aligned}
$$

We note that

$$
\frac{S_{t}}{S_{t_{i}}} H_{t_{i}}^{(D, 2)}=K_{t_{i}}\left(\exp (y)-\tilde{K}_{t_{i}}\right)^{+}
$$

where $\tilde{K}_{t_{i}}$ is as in (4.37) and $y=\log \left(S_{t} / S_{t_{i}}\right)$. Applying Lemma 1 , with $w>1$, and making use of Fubini's theorem, the second part of (4.40) is

$$
V_{t, t_{i}}^{(D, 2 b)}=(1-\rho)^{i-1-j} K_{t_{i}} \frac{1}{2 \pi} \int_{\mathbb{R}} \mathcal{E}_{t, t_{i}}^{(A)} \frac{\tilde{K}_{t_{i}}^{-(w-1+\iota \lambda)}}{(w+\iota \lambda)(w-1+\iota \lambda)} d \lambda,
$$

and the expectation inside the integral can be evaluated using Proposition 3. Similarly, the first part of (4.40) is

$$
V_{t, t_{i}}^{(D, 2 a)}=(1-\rho)^{i-1-j} K_{t_{i}} \frac{1}{2 \pi} \int_{\mathbb{R}} \mathcal{E}_{t, t_{i}}^{(D)} \frac{\tilde{K}_{t_{i}}^{-(w-1+\iota \lambda)}}{(w+\iota \lambda)(w-1+\iota \lambda)} d \lambda .
$$

We note that $V_{t, t_{n}}^{(A, 1)}$ and $V_{t, t_{n}}^{(A, 2)}$ are evaluated in similar ways to those done for $V_{t, t_{i}}^{(D, 1)}$ and $V_{t, t_{i}}^{(D, 2)}$, respectively. Finally, noting that

$$
\frac{S_{t}}{S_{t_{n}}} H_{t_{n}}^{(A, 3)}=\left(K_{t_{n}}\right)^{3}\left(\tilde{\tilde{K}}_{t_{n}}-\exp (y)\right)^{+},
$$

where $\tilde{\tilde{K}}_{t_{n}}$ is as in (4.37) and $y=\log \left(S_{t} / S_{t_{n}}\right)$, we have, upon applying Lemma 1 , with $w<0$,

$$
V_{t, t_{n}}^{(A, 3)}=(1-\rho)^{n-j}\left(K_{t_{n}}\right)^{3} \frac{1}{2 \pi} \int_{\mathbb{R}} \mathcal{E}_{t, t_{n}}^{(A)} \frac{\left(\tilde{\tilde{K}}_{t_{n}}\right)^{-(w-1+\iota \lambda)}}{(w+\iota \lambda)(w-1+\iota \lambda)} d \lambda,
$$

and the expectation inside the integral can be evaluated using Theorem 3 .

Remark 2. We note that the expression for $V^{(D, 1)}$ equals the investment account today, diminished by the lapses and management fees incurred up to the payment time $t_{i}$. Also, the expressions for $V^{(D, 2 a)}$ and $V^{(D, 2 b)}$ combine to give the put option component of the death benefit. The expression for $V^{(A, 1)}$ equals the survival probability, diminished by lapses and management fees incurred up to the maturity time $t_{n}$. Finally, the expressions for $V^{(A, 2)}$ and $V^{(A, 3)}$ correspond to the integral transforms of the put option and call option payoffs respectively.

We have the following corollary, when $r, \bar{S}$ and $\mu$ are driven by independent Wiener processes, i.e. $\alpha^{r}=\alpha^{\mu}=\beta^{\mu}=0$. 
Corollary 1. The real-world values of the components of the death and accumulation benefits are

$$
V_{t, t_{i}}^{(D, 1)}=(1-\rho)^{i-1-j} \frac{S_{t}}{S_{t_{0}}} \exp \left\{-\xi\left(t_{i}-t_{0}\right)\right\}\left({ }_{i-j-1} \hat{p}_{x+t_{j}}\left(t_{j}\right)-{ }_{i-j} \hat{p}_{x+t_{j}}\left(t_{j}\right)\right)
$$

$$
V_{t, t_{i}}^{(D, 2)}=(1-\rho)^{i-1-j}{ }_{i-j-1} \hat{p}_{x+t_{j}}\left(t_{j}\right) \hat{q}_{x+t_{i-1}}\left(t_{j}\right) \frac{1}{S_{t_{0}}} \exp \left\{-\xi\left(t_{i}-t_{0}\right)\right\} p_{t_{i}, K_{t_{i}}^{\prime}}\left(t, S_{t}\right)
$$

$$
V_{t, t_{n}}^{(A, 1)}=(1-\rho)^{n-j}{ }_{n-j} \hat{p}_{x+t_{j}}\left(t_{j}\right) \frac{S_{t}}{S_{t_{0}}} \exp \left\{-\xi\left(t_{n}-t_{0}\right)\right\}
$$

$$
V_{t, t_{n}}^{(A, 2)}=(1-\rho)^{n-j}{ }_{n-j} \hat{p}_{x+t_{j}}\left(t_{j}\right) \frac{1}{S_{t_{0}}} \exp \left\{-\xi\left(t_{n}-t_{0}\right)\right\} p_{t_{i}, K_{t_{n}}^{\prime}}\left(t, S_{t}\right)
$$

$$
V_{t, t_{n}}^{(A, 3)}=(1-\rho)^{n-j}{ }_{n-j} \hat{p}_{x+t_{j}}\left(t_{j}\right) \frac{1}{S_{t_{0}}} \exp \left\{-\xi\left(t_{n}-t_{0}\right)\right\} c_{t_{i}, K_{t_{n}}^{\prime \prime}}\left(t, S_{t}\right),
$$

where $K_{t_{i}}^{\prime}$ and $K_{t_{n}}^{\prime \prime}$ are as in (4.57) and where we define the put and call option values

$$
p_{T, K}\left(t, S_{t}\right)=E_{t}\left(\frac{S_{t}}{S_{T}}\left(K-S_{T}\right)^{+}\right), \quad c_{T, K}\left(t, S_{t}\right)=E_{t}\left(\frac{S_{t}}{S_{T}}\left(S_{T}-K\right)^{+}\right) .
$$

Thus, we have demonstrated how the death and accumulation benefits with guarantees can be evaluated using Theorem 6, Corollary 1 and the two aggregation equations $V_{t, t_{i}}^{(D)}=V_{t, t_{i}}^{(D, 1)}+V_{t, t_{i}}^{(D, 2)}$ and $V_{t, t_{n}}^{(A)}=V_{t, t_{n}}^{(A, 1)}+V_{t, t_{n}}^{(A, 2)}-V_{t, t_{n}}^{(A, 3)}$.

4.3. Valuation Formulae for Variable Annuities. We are in a position to provide a valuation formula for the variable annuity specified earlier in Section 2, whose payoffs are given in (2.1), (2.3) and (2.4). We recall from Section 2 the notation $\rho$ for the lapse rate and $\xi$ for the management fee, and from Section 4 the notation $\hat{q}_{x}(t)$ for the realised one-year death rate of a life aged $x$ at time $t$ and $\hat{p}_{x}(t)$ for the realised one-year survival rate of a life aged $x$ at time $t$. In the light of Theorems 3 and 6 , the value of our VA product specified in Section 2 is computed as

$$
V_{t}^{R W}=\left\{\sum_{i=j+1}^{n}\left(V_{t, t_{i}}^{(D)}+V_{t, t_{i}}^{(L)}\right)\right\}+V_{t, t_{n}}^{(A)},
$$

and, in the instance when $\alpha^{r}=\alpha^{\mu}=\beta^{\mu}=0$ and therefore when $r, \bar{S}$ and $\mu$ are independent processes, the formula for the real-world VA policy value, based on policies in force at the start of the year,

$$
\begin{aligned}
V_{t}^{R W} & =\sum_{i=j+1}^{n}{ }_{i-(j+1)} \hat{p}_{x+j}\left(t_{j}\right)(1-\rho)^{i-j-1}\left\{\hat{q}_{x+i-1}\left(t_{j}\right) V_{t, t_{i}}^{R W}+\rho\left(1-\hat{q}_{x+i-1}\left(t_{j}\right)\right) S V_{t, t_{i}}^{R W}\right\} \\
& +{ }_{n-j} \hat{p}_{x+j}\left(t_{j}\right)(1-\rho)^{n-j} A V_{t, t_{n}}^{R W},
\end{aligned}
$$


where $t \in\left[t_{j}, t_{j+1}\right)$, the death benefit value $V_{t, t_{i}}^{R W}$ is

$$
V_{t, t_{i}}^{R W}=\frac{S_{t}}{S_{t_{0}}} \exp \left\{-\xi\left(t_{i}-t_{0}\right)\right\}+\frac{1}{S_{t_{0}}} \exp \left\{-\xi\left(t_{i}-t_{0}\right)\right\} p_{t_{i}, K_{t_{i}}^{\prime}}\left(t, S_{t}\right),
$$

the surrender benefit value $S V_{t, t_{i}}^{R W}$ is

$$
S V_{t, t_{i}}^{R W}=\sqrt{K_{t_{i}}} P\left(t, t_{i}\right)
$$

and the survival benefit value $A V_{t, t_{i}}^{R W}$ is

$$
\begin{aligned}
A V_{t, t_{n}}^{R W} & =\frac{S_{t}}{S_{t_{0}}} \exp \left\{-\xi\left(t_{n}-t_{0}\right)\right\} \\
& +\frac{1}{S_{t_{0}}} \exp \left\{-\xi\left(t_{n}-t_{0}\right)\right\} p_{t_{n}, K_{t_{n}}^{\prime}}\left(t, S_{t}\right) \\
& -\frac{1}{S_{t_{0}}} \exp \left\{-\xi\left(t_{n}-t_{0}\right)\right\} c_{t_{n}, K_{t_{n}}^{\prime \prime}}\left(t, S_{t}\right),
\end{aligned}
$$

with

$$
\begin{aligned}
K_{t_{i}}^{\prime} & =S_{t_{0}} \exp \left\{\xi\left(t_{i}-t_{0}\right)\right\} K_{t_{i}} \\
K_{t_{n}}^{\prime \prime} & =S_{t_{0}} \exp \left\{\xi\left(t_{n}-t_{0}\right)\right\}\left(K_{t_{n}}\right)^{3} .
\end{aligned}
$$

Here $p_{T, K}\left(t, S_{t}\right)$ and $c_{T, K}\left(t, S_{t}\right)$ denote the real-world values of a put option and call option respectively, given in (4.51).

Analogous formulae are straightforwardly obtained for the corresponding riskneutral valuations.

\section{Description of Hedging Methodology}

In addition to the valuation of long-dated VAs, our aim is to demonstrate lessexpensive hedging such policies. In respect of the VA, a hedging strategy is a trading strategy involving a portfolio of hedge securities whose value at each of the prescribed payoff dates is intended to replicate the value of the VA.

5.1. Hedge Portfolio. Because the models considered in this paper are composed of a stochastic short rate, a stochastic discounted GOP and a stochastic mortality rate and because our universe of securities consists of cash, bonds, stock index and the variable annuity, we have two hedgeable random factors which can be hedged using a managed self-financing portfolio of cash $B_{t}$, the GOP index $S_{t}$ and a tenyear coupon bond $F_{t}$. The value at time $t \geq 0$ of the hedge portfolio $\pi$ can be written as

$$
V_{t}^{(\pi)}=\delta_{t}^{B} B_{t}+\delta_{t}^{S} S_{t}+\delta_{t}^{F} F_{t},
$$

where $\delta^{B}, \delta^{S}$ and $\delta^{F}$ are the numbers of units of the savings account, GOP index and ten-year coupon bond, respectively, as specified in (5.4).

5.2. Cost and Benchmarked Cost of Hedging. The cost $C_{t}$ at time $t$ of hedging a derivative since initial time 0 is equal to the cost of the derivative at time $t$ less any gains from trading the hedge portfolio. We write

$$
C_{t}=V_{t}^{H}-\int_{0}^{t} \delta_{u}^{B} d B_{u}-\int_{0}^{t} \delta_{u}^{S} d S_{u}-\int_{0}^{t} \delta_{u}^{F} d F_{u}=V_{t}^{H}-\int_{0}^{t} d V_{u}^{(\pi)}
$$


where $V_{t}^{H}$ is the value of the derivative at time $t$ and $V_{t}^{(\pi)}$ is the value of the hedge portfolio at time $t$.

We demonstrate the efficacy of reserving for a large number of policies under each of our considered models, by performing a backtest of the reserving strategy, where we forecast the mortality rates using either (3.19) or (3.20) assume that the realised mortality rates of policyholders are those of the population. In practice, insurers underwrite their policies to classify the risks being insured, and this means that, in general, the mortality of the policyholders will differ from that of the population. This difference in mortality is termed level mortality risk and in this paper we are assuming this is zero. Furthermore, insurers will only have a finite number of policies, which means that even if the insurer knows the mortality rates of policyholders, their realised rates will differ. This difference in mortality is termed stochastic mortality risk and in this paper we are assuming this is zero, although insurers will write sufficiently many policies to diversify away this risk.

Denoting the initial reserves held in respect of all policies in force at time $t_{0}$ by $R_{t_{0}}^{R W, \pi}$, we set

$$
R_{t_{0}}^{R W, \pi}=V_{t_{0}}^{R W}
$$

implicitly assuming a notional premium amount equal to one unit for the entirety of policyholders. Employing delta hedging at discrete times in the set $G^{\prime}=\left\{t_{0}, t_{0}+\right.$ $\left.\Delta, \ldots, t_{1}, \ldots, t_{n}\right\}$, we apportion the reserves among the savings account, GOP and ten-year bond as

$$
R_{t_{0}}^{R W, \pi}=\delta_{t_{0}}^{B} B_{t_{0}}+\delta_{t_{0}}^{S} S_{t_{0}}+\delta_{t_{0}}^{F} F_{t_{0}}
$$

where, for $t \in G^{\prime}$,

$$
\begin{aligned}
\delta_{t}^{F} & =\frac{\partial R_{t}^{R W}}{\partial r_{t}} / \frac{\partial F_{t}^{R W}}{\partial r_{t}}, \\
\delta_{t}^{S} & =\frac{\partial R_{t}^{R W}}{\partial S_{t}}-\delta_{t}^{F} \frac{\partial F_{t}^{R W}}{\partial S_{t}}, \\
\delta_{t}^{B} & =\left\{R_{t}^{R W, \pi}-\delta_{t}^{S} S_{t}-\delta_{t}^{F} F_{t}\right\} / B_{t},
\end{aligned}
$$

and $R_{t}^{R W}$ denotes the value of in-force policies at time $t$, given by

$$
R_{t}^{R W}={ }_{j} p_{x}\left(t_{0}\right)(1-\rho)^{j} V_{t}^{R W}
$$

for $t \in\left[t_{j}, t_{j+1}\right)$.

Reserves at Subsequent Times

Bearing in mind that we are reserving for the portfolio of VA policies, and that at subsequent times some of the policyholders will have either died or lapsed, we value the in-force policy portfolio at time $t \in\left[t_{j}, t_{j+1}\right)$ as per (5.5). Also, the sensitivity of the in-force policy portfolio value with respect to $S_{t}$, and by analogy for the sensitivity with respect to $r_{t}$, is computed as

$$
\begin{aligned}
\frac{\partial R_{t}^{R W}}{\partial S_{t}} & ={ }_{j} p_{x}\left(t_{0}\right)(1-\rho)^{j} \sum_{i=j+1}^{n}\left[{ }_{i-(j+1)} \hat{p}_{x+j}\left(t_{j}\right)(1-\rho)^{i-j-1}\right. \\
& \left.\times\left\{\hat{q}_{x+i-1}\left(t_{j}\right) \frac{\partial V_{t, t_{i}}^{R W}}{\partial S_{t}}+\rho\left(1-\hat{q}_{x+i-1}\left(t_{j}\right)\right) \frac{\partial S V_{t, t_{i}}^{R W}}{\partial S_{t}}\right\}\right] \\
& +{ }_{n-j} \hat{p}_{x+j}\left(t_{j}\right)(1-\rho)^{n-j} \frac{\partial A V_{t, t_{n}}^{R W}}{\partial S_{t}}
\end{aligned}
$$




\begin{tabular}{cccc}
\hline SR Model & Mortality Model & BS Value & MMM Value \\
\hline Vasicek & RW & 0.8589 & 0.8445 \\
Vasicek & OU & 0.8220 & 0.8026 \\
CIR & RW & 0.8589 & 0.8444 \\
CIR & OU & 0.8238 & 0.8038 \\
$3 / 2$ & RW & 0.8688 & 0.8275 \\
$3 / 2$ & OU & 0.8335 & 0.7877 \\
\hline
\end{tabular}

TABLE 7. Initial policy values under each of the models.

where, for $i \in\{j+1, \ldots, n\}$, the deltas

$$
\frac{\partial V_{t, t_{i}}^{R W}}{\partial S_{t}}, \frac{\partial S V_{t, t_{i}}^{R W}}{\partial S_{t}}, \frac{\partial A V_{t, t_{i}}^{R W}}{\partial S_{t}}, \frac{\partial V_{t, t_{i}}^{R W}}{\partial r_{t}}, \frac{\partial S V_{t, t_{i}}^{R W}}{\partial r_{t}}, \frac{\partial A V_{t, t_{i}}^{R W}}{\partial r_{t}}
$$

are computed using numerical approximations.

At the next hedging time $t_{0}+\Delta$ our reserves become

$$
R_{t_{0}+\Delta}^{R W, \pi}=\delta_{t_{0}}^{B} B_{t_{0}+\Delta}+\delta_{t_{0}}^{S} S_{t_{0}+\Delta}+\delta_{t_{0}}^{F} F_{t_{0}+\Delta}
$$

and we rebalance our portfolio with $\delta_{t}^{B}, \delta_{t}^{S}$ and $\delta_{t}^{F}$ computed according to (5.4).

We proceed in this manner until we arrive at a payment date $t_{i} \in\left\{t_{1}, t_{2}, \ldots, t_{n}\right\}$ where we deduct from the accumulated reserves the realised probability-weighted payoffs due to death and lapsation, giving

$$
\begin{aligned}
R_{t_{i}}^{R W, \pi} & =\delta_{t_{i}-\Delta}^{B} B_{t_{i}}+\delta_{t_{i}-\Delta}^{S} S_{t_{i}}+\delta_{t_{i}-\Delta}^{F} F_{t_{i}} \\
& -{ }_{i-1} p_{x}\left(t_{0}\right)(1-\rho)^{i-1}\left\{q_{x+i-1}\left(t_{i-1}\right) H_{t_{i}}^{(D)}+p_{x+i-1}\left(t_{i-1}\right) \rho H_{t_{i}}^{(L)}\right\} .
\end{aligned}
$$

At time $t_{n}$ we also deduct the payoff in respect of the accumulation benefit.

5.3. Hedging Results for Variable Annuity. In this subsection, we provide the results of hedging our variable annuity, for the case of policies issued to lives aged 65 in 1971 with term 45 years, namely the case $t_{0}=1971, x=65$ and $n=45$. Using the data sets and parameter values mentioned earlier, we have the initial policy values under each of the models given in Table 7. It is evident that MMM models of the discounted GOP lead to lower initial policy values.

Figure 3 illustrates the policy values and reserving strategies under the BS and MMM models, with random walk mortality and Vasicek short rate, where the surplus levels of reserves attained by the strategy in respect of the MMM are evident in the latter years of the policy, despite having lower initial reserves. These surpluses generated via the MMM strategy are helpful when actual mortality and lapse rates vary from those assumed in our models, and demonstrate the less-expensive reserving method proposed. Figures 9, 10 and 11 in Appendix C illustrate these phenomena when the assumptions for the management fee, roll-up rate of the guarantee, lapse rate and the factor applied to the roll-up rate for the surrender benefit are varied.

The less-expensive reserving strategy of the MMM can be attributed particularly to less-expensive values of zero-coupon bonds and less-expensive values of put options. The less-expensive values of zero-coupon bonds under the MMM are evident from a comparison of (4.11) with (4.12). The less-expensive values of put options under the MMM are not so clear from the formulae supplied earlier and we therefore demonstrate this in the following subsection. 


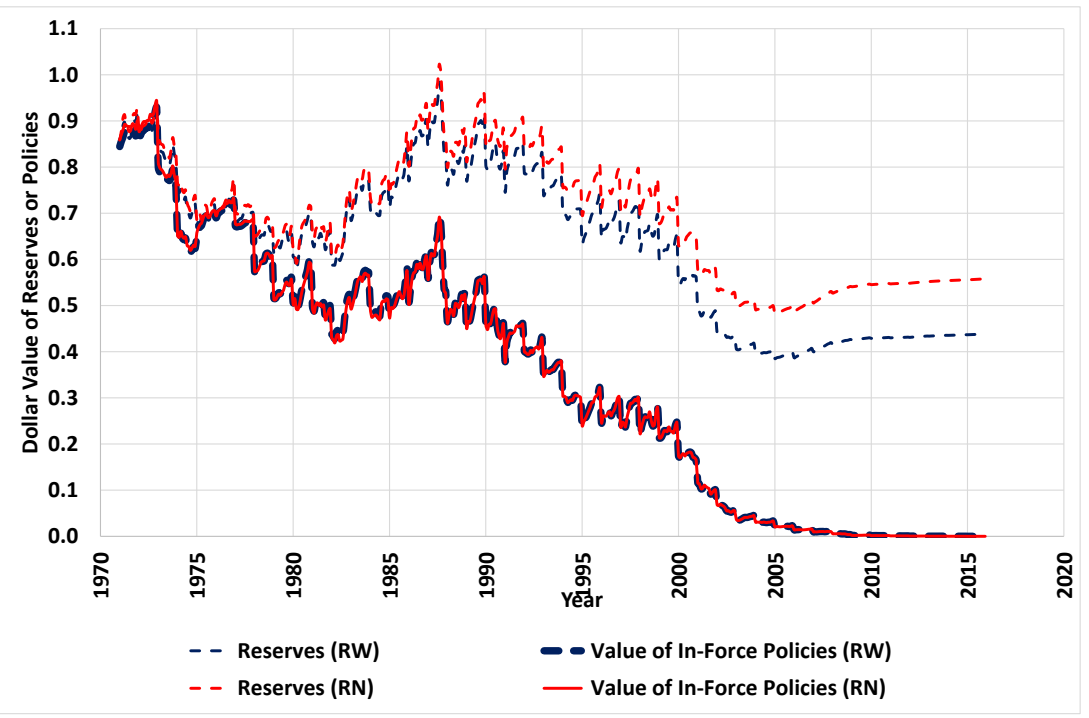

FiguRE 3. Reserves and policy values under the MMM and the Black-Scholes model, with random walk mortality and Vasicek short rate, of the variable annuity with guaranteed minimum death benefit over the period from 1971 until 2016, which provides annual payments from 1972 until 2016.

5.4. Hedging Results for Put Option. In this subsection, we focus on hedging a long-dated put option expiring at time $T$ whose strike price $K$ keeps pace with the level of the equity index by way of the formula

$$
K=S_{t_{0}} \exp \left(\left(\eta+m_{r}\right)\left(T-t_{0}\right)\right),
$$

where $t_{0}$ is the time at which the put option is written and $\eta=0.046841$ is the net market growth rate and $m_{r}=\frac{1}{141} \sum_{s} r(s)=0.045726$ is the average of the one year continuously compounded cash rates over the 140 year period of the data. As per the real-world valuation formula (A.1), the time-t value of the put option is

$$
V_{t}=S_{t} E_{t}\left(\frac{1}{S_{T}}\left\{S_{T}-S_{t} \exp \left(\left(\eta+m_{r}\right)\left(T-t_{0}\right)\right)\right\}^{+}\right) .
$$

We attempt to replicate the terminal value of the put option using delta hedging and, using (5.2), compute the benchmarked costs of hedging a put option at expiry over all possible periods within the data set. From this the 99-th percentile of the set of benchmarked costs is computed, shown in Table 8 and Figure 4. We determine the best market model to be the one for which there corresponds the minimum percentile benchmarked cost of hedging.

Each model for which the discounted GOP is modelled by the MMM has significantly lower costs of hedging long-dated put options and thus lower costs of hedging 


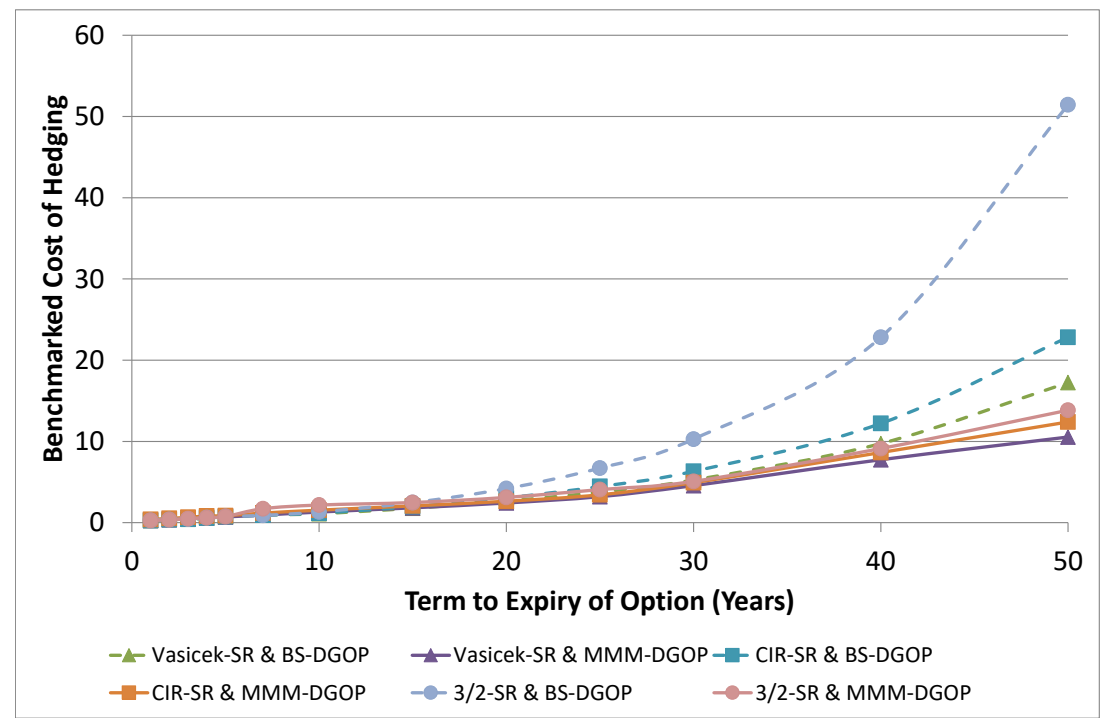

Figure 4. Graph showing the 99-th percentile cost of hedging put options of varying terms to expiry and corresponding strike prices given in (5.10).

\begin{tabular}{ccccccc}
\hline $\begin{array}{c}\text { Term } \\
\text { (Years) }\end{array}$ & $\begin{array}{c}\text { Vasicek \& } \\
\text { BS }\end{array}$ & $\begin{array}{c}\text { Vasicek \& } \\
\text { MMM }\end{array}$ & $\begin{array}{c}\text { CIR \& } \\
\text { BS }\end{array}$ & $\begin{array}{c}\text { CIR \& } \\
\text { MMM }\end{array}$ & $\begin{array}{c}3 / 2 \& \text { BS } \\
\text { BS }\end{array}$ & $\begin{array}{c}3 / 2 \& \\
\text { MMM }\end{array}$ \\
\hline 1 & 0.26 & 0.27 & 0.26 & 0.39 & 0.27 & 0.27 \\
2 & 0.39 & 0.35 & 0.38 & 0.52 & 0.38 & 0.35 \\
3 & 0.42 & 0.47 & 0.43 & 0.65 & 0.44 & 0.48 \\
4 & 0.57 & 0.60 & 0.58 & 0.82 & 0.61 & 0.60 \\
5 & 0.74 & 0.70 & 0.74 & 0.85 & 0.75 & 0.75 \\
7 & 0.92 & 0.93 & 0.92 & 1.18 & 0.89 & 1.71 \\
10 & 1.10 & 1.31 & 1.17 & 1.54 & 1.32 & 2.17 \\
15 & 1.77 & 1.82 & 1.97 & 2.06 & 2.47 & 2.47 \\
20 & 2.65 & 2.42 & 3.05 & 2.63 & 4.19 & 3.10 \\
25 & 3.77 & 3.20 & 4.45 & 3.43 & 6.72 & 4.07 \\
30 & 5.25 & 4.56 & 6.31 & 4.92 & 10.28 & 5.08 \\
40 & 9.73 & 7.74 & 12.21 & 8.63 & 22.82 & 9.12 \\
50 & 17.24 & 10.54 & 22.83 & 12.39 & 51.44 & 13.84 \\
\hline
\end{tabular}

TABLE 8. 99-th percentile costs of hedging put options of varying terms to expiry for each combination of short rate and discounted GOP models.

the guaranteed accumulation benefit embedded in the variable annuity. In particular, we find that among the models having a stochastic short rate, the Vasicek 
short rate and MMM discounted GOP model provides the best hedging strategy for long-dated GOP put options.

\section{Conclusion}

The paper illustrates the application of the real-world valuation methodology under the benchmark approach, which is more general than what is given under the classical risk-neutral approach, and using a modelling framework that has dependence between the stock market, interest rates and mortality rates. The presented approach is more general than the risk neutral approach because the requirement that an equivalent martingale probability measure exists is not needed, allowing, for example, the discounted GOP to be modelled as in the MMM.

Under the assumption of independence of mortality, the equity index and shortterm interest rates the backtesting of valuations and reserving strategies under various models has been performed, allowing a comparison under both real-world and classical valuation approaches.

When the discounted GOP is modelled as in the MMM, the benchmark approach permits the better long-term performance of the equity market compared to that of

the fixed income market to be systematically exploited to produce payoffs in respect of variable annuities in a less-expensive way than done by classical approaches.

\section{REFERENCES}

D.H. Ahn and B. Gao. A parametric nonlinear model of term structure dynamics. Review of Financial Studies, 12(4):721-762, 1999.

Y. Ait-Sahalia. Nonparametric pricing of interest rate derivative securities. Econometrica, 64:527-560, 1996.

A. R. Bacinello, P. Millossovich, A. Olivieri, and E. Pitacco. Variable annuities: A unifying valuation approach. Insurance: Mathematics and Economics, 49(3): 285-297, 2011.

J. Baldeaux and E. Platen. Functionals of Multidimensional Diffusions with Applications to Finance. Bocconi University Press \& Springer, New York, 2013.

D. Bauer, A. Kling, and J. Russ. A universal pricing framework for guaranteed minimum benefits in variable annuities. ASTIN Bulletin, 38(2):621-651, 2008.

F. Black and M. Scholes. The pricing of options and corporate liabilities. Journal of Political Economy, 81:637-654, 1973.

P. Boyle and M. Hardy. Guaranteed annuity options. ASTIN Bulletin, 33(2): 125-152, 2003.

K. C. Chan, G. A. Karolyi, and R. M. Stulz. Global financial markets and the risk premium on u.s. equity markets. Journal of Financial Economics, 32:137-67, 1992.

J. C. Cox, J. E. Ingersoll, and S. A. Ross. A theory of the term structure of interest rates. Econometrica, 53(2):385-407, 1985.

M. Craddock and K. Lennox. The calculation of expectations for classes of diffusion processes by Lie symmetry methods. The Annals of Applied Probability, 19(1): 127-157, 2009.

M. Dahl and T. Moller. Valuation and hedging of life insurance liabilities with systematic mortality risk. Insurance: Mathematics and Economics, 39:193-217, 2006. 
F. Delbaen and W. Schachermayer. A general version of the fundamental theorem of asset pricing. Mathematische Annalen, 300(3):463-520, 1994.

D. Duffie and R. Kan. A yield-factor model of interest rates. Mathematical Finance, 6:379-406, 1996.

M. Escobar, M. Krayzler, F. Ramsauer, D. Saunders, and R. Zagst. Incorporation of stochastic policyholder behavior in analytical pricing of GMABs and GMDBs. Risks, 4(4):41, 2016. URL https://doi.org/10.3390/risks4040041.

K. Fergusson. Asymptotics of bond yields and volatilities for extended Vasicek models under the real-world measure. Annals of Financial Economics, 12(1), 2017a.

K. Fergusson. Explicit formulae for parameters of stochastic models of a discounted equity index using maximum likelihood estimation with applications. Annals of Financial Economics, 12(2):1-31, 2017b. doi: 10.1142/S2010495217500105.

K. Fergusson and E. Platen. Less-expensive pricing and hedging of long-dated equity index options when interest rates are stochastic. Research Paper Series 357, Quantitative Finance Research Centre, University of Technology, Sydney, 2015a.

K. Fergusson and E. Platen. Application of maximum likelihood estimation to stochastic short rate models. Annals of Financial Economics, 10(2), 2015b.

D. Filipović. Term Structure Models: A Graduate Course. Springer-Verlag, 2009.

B. Gompertz. On the nature of the function expressive of the law of human mortality, and on a new mode of determining the value of life contingencies. Philosophical Transactions of the Royal Society of London, 115:513-583, 1825.

S. Haithcock. The costs of owning an annuity. American Association of Individual Investors, September 2013, 2013.

J. Hartman. 2017 variable annuity guaranteed benefits survey. Society of Actuaries, 2018.

H. Hulley and E. Platen. Hedging for the long run. Research Paper Series 214, Quantitative Finance Research Centre, University of Technology, Sydney, 2008.

I. Karatzas and S. E. Shreve. Brownian Motion and Stochastic Calculus. Springer Verlag, second edition, 1991.

R. D. Lee and L. R. Carter. Modeling and forecasting U.S. mortality. Journal of the American Statistical Association, 87(419):659-671, 1992.

W. M. Makeham. On the law of mortality and the construction of annuity tables. The Assurance Magazine and the Journal of the Institute of Actuaries, 8(6):301$310,1860$.

T. Marquardt, E. Platen, and S. Jaschke. Valuing guaranteed minimum death benefit options in variable annuities under a Benchmark Approach. University of Technology, Sydney Research Paper 221, 2008.

M. A. Milevsky and S. E. Posner. The Titanic option: Valuation of the guaranteed minimum death benefit in variable annuities and mutual funds. Journal of Risk and Insurance, 68(1):93-128, 2001.

C. Pintoux and N. Privault. The Dothan pricing model revisited. Mathematical Finance, 21:355-363, 2011.

E. Platen. A short-term interest rate model. Finance and Stochastics, 3:215-225, 1999.

E. Platen. A minimal financial market model. In Trends in Mathematics, pages 293-301. Birkhäuser, 2001. 
E. Platen. Diversified portfolios with jumps in a benchmark framework. AsiaPacific Financial Markets, 11(1):1-22, 2005.

E. Platen and D. Heath. A Benchmark Approach to Quantitative Finance. Springer Finance, 2006.

E. Platen and R. Rendek. Approximating the numéraire portfolio by naive diversification. Journal of Asset Management, 13(1):34-50, 2012.

D. Schrager. Affine stochastic mortality. Insurance: Mathematics and Economics, 38:81-97, 2006.

R. Shiller. Market Volatility. The MIT Press, Cambridge, Massachusetts, 1989.

W. Siler. A competing-risk model for animal mortality. Ecology, 60:750-757, 1979.

W. Siler. Parameters of mortality in human populations with widely varying life spans. Statistics in Medicine, 2:373-380, 1983.

T.N. Thiele. On a mathematical formula to express the rate of mortality throughout the whole of life, tested by a series of observations made use of by the Danish Life Insurance Company of 1871. Journal of the Institute of Actuaries and Assurance Magazine, 16(5):313-329, 1871.

E. Ulm. Analytic solution for return of premium and rollup guaranteed minimum death benefit options under some simple mortality laws. ASTIN Bulletin, 38(2): 543-563, 2008.

A. van Haastrecht, R. Lord, A. Pelsser, and D. Schrager. Pricing long-dated insurance contracts with stochastic interest rates and stochastic volatility. Insurance: Mathematics and Economics, 45:436-448, 2009.

O. A. Vasicek. An equilibrium characterization of the term structure. Journal of Financial Economics, 5:177-188, 1977.

X. Zhu, M. R. Hardy, and D. Saunders. Dynamic hedging strategies for cash balance pension plans. ASTIN Bulletin, 48(3):1245-1275, 2018.

\section{Appendix A. Valuation of Contingent Claims Under the Benchmark APPROACH}

We provide here a summary of the benchmark approach to valuation. Consider a financial market driven by $d$-dimensional Brownian motion $\mathbf{W}=\left\{\mathbf{W}_{t}: t \geq 0\right\}$ defined on a filtered probability space $(\Omega, \mathcal{A}, \underline{\mathcal{A}}, P)$, where the filtration $\underline{\mathcal{A}}$ is right continuous and complete. Assume there are a locally riskless savings account process $S^{0}$, such that $d S_{t}^{0}=r_{t} S_{t}^{0} d t$ for an interest rate process $r$, and $m$ primary security accounts, corresponding to stocks, currencies, bonds and commodities each denominated in the currency of the savings account, whose values at time $t$ are $\mathbf{S}_{t} \in \mathbb{R}^{m}$ such that $d S_{t}^{j}=S_{t}^{j}\left\{\left(r_{t}+\mu_{t}^{j}\right) d t+\left(\mathbf{b}_{t}^{j}\right)^{\top} d \mathbf{W}_{t}\right\}$, where $\boldsymbol{\mu}_{t}=\left(\mu_{t}^{1}, \ldots, \mu_{t}^{m}\right)^{\top}$ is the drift vector, $\mathbf{b}_{t}^{j} \in \mathbb{R}^{d}$ and $\mathbf{B}_{t}=\left(\mathbf{b}_{t}^{1}, \ldots, \mathbf{b}_{t}^{m}\right)^{\top}$ is the volatility matrix. The $j$-th primary security account, for $j \in\{0,1,2, \ldots, m\}$, represents the accumulation of all income, costs of carry plus capital gains and losses achieved while holding the underlying primary security. We restrict ourselves to the case $m=d$ so that our financial market is complete. When $m>d$ we have redundant securities which can be removed from the set of primary security accounts. Alternatively, if $m<d$ then the financial market is incomplete. Let $S^{\pi}$ be a self-financing portfolio process with weights $\pi_{t}^{1}, \ldots, \pi_{t}^{m}$ in primary security accounts and the remaining weight $1-\boldsymbol{\pi}_{t}^{\top} \mathbf{1}$ in the savings account, so that $d \log S_{t}^{\boldsymbol{\pi}}=\left\{r_{t}+\boldsymbol{\pi}_{t}^{\top} \boldsymbol{\mu}_{t}-\frac{1}{2} \boldsymbol{\pi}_{t}^{\top}\left(\mathbf{B}_{t} \mathbf{B}_{t}^{\top}\right) \boldsymbol{\pi}_{t}\right\} d t+\boldsymbol{\pi}_{t}^{\top} \mathbf{B}_{t} d \mathbf{W}_{t}$. The growth optimal portfolio (GOP) is the unique portfolio $S^{\pi^{*}} \equiv S$ that makes 
any benchmarked portfolio process $\hat{S}^{\boldsymbol{\pi}} \equiv S^{\boldsymbol{\pi}} / S$ a local martingale. It is this supermartingale property which is connected with the absence of strong arbitrage in our financial market, namely the inability to generate strictly positive profits in finite time with zero initial wealth. Furthermore, the absence of strong arbitrage does not preclude the existence of classical arbitrage opportunities, but in instances where classical arbitrage is precluded, agreement between the benchmark approach to valuation and risk-neutral valuation is attained. Assuming for Lebesgue-almost every $t$ invertibility of $\mathbf{B}_{t} \mathbf{B}_{t}^{\top}$, we have that $\boldsymbol{\pi}^{*}{ }_{t}=\left(\mathbf{B}_{t} \mathbf{B}_{t}^{\top}\right)^{-1} \boldsymbol{\mu}_{t}$ and letting $\boldsymbol{\theta}_{t}=\mathbf{B}_{t}^{\top} \boldsymbol{\pi}_{t}^{*}$ we can write the SDE for the GOP as $d S_{t}=S_{t}\left(r_{t}+\left\|\boldsymbol{\theta}_{t}\right\|^{2}\right) d t+S_{t} \boldsymbol{\theta}_{t}^{\top} d \mathbf{W}_{t}$. We remark that invertibility of the volatility matrix $\mathbf{B}_{t} \mathbf{B}_{t}^{\top}$ ensures that the market price of risk vector $\boldsymbol{\theta}_{t}=\mathbf{B}_{t}^{\top}\left(\mathbf{B}_{t} \mathbf{B}_{t}^{\top}\right)^{-1} \boldsymbol{\mu}_{t}$ is unique. Defining the process $W$ via $d W_{t}=\boldsymbol{\theta}_{t}^{\top} d \mathbf{W}_{t} /\left\|\boldsymbol{\theta}_{t}\right\|$, we have that $W$ is a Brownian motion and, therefore, the we can rewrite the SDE for $S$ as $d S_{t}=S_{t}\left(r_{t}+\left\|\boldsymbol{\theta}_{t}\right\|^{2}\right) d t+S_{t}\left\|\boldsymbol{\theta}_{t}\right\| d W_{t}$ and the SDE for the discounted GOP $\bar{S}=S / S^{0}$ as $d \bar{S}_{t}=\bar{S}_{t}\left\|\boldsymbol{\theta}_{t}\right\|^{2} d t+\bar{S}_{t}\left\|\boldsymbol{\theta}_{t}\right\| d W_{t}$. Another form of the SDE for the discounted GOP results, if we define $\alpha_{t}=\bar{S}_{t}\left\|\boldsymbol{\theta}_{t}\right\|^{2}$, namely $d \bar{S}_{t}=\alpha_{t} d t+\sqrt{\alpha_{t} \bar{S}_{t}} d W_{t}$. For a given contingent claim $H_{T}$ payable at time $T \in(0, \infty)$, it has been shown in Platen and Heath [2006] that the minimal possible value $V_{t}^{H}$ for a replicating hedge portfolio satisfies the real-world valuation formula

$$
V_{t}^{H}=E_{t}\left(\frac{S_{t}}{S_{T}} H_{T}\right)
$$

where $E_{t}$ denotes the real-world conditional expectation under the real-world probability measure. The GOP $S$ is taken here as the numéraire or benchmark and the benchmarked value

$$
\hat{V}_{t}^{H}=\frac{V_{t}^{H}}{S_{T}}
$$

forms a martingale when assuming its value to be finite. When an equivalent risk neutral probability measure exists, the candidate Radon-Nikodym derivative process $\Lambda=\left\{\Lambda_{t}: t \in[0, T]\right\}$, given by

$$
\Lambda_{t}=\left.\frac{d Q}{d P}\right|_{\mathcal{A}_{t}}=\frac{S_{t}^{0}}{S_{0}^{0}} \frac{S_{0}}{S_{t}}
$$

is a martingale and we have

$$
V_{t}^{H}=E_{t}\left(\frac{S_{t}}{S_{T}} H_{T}\right)=E_{t}\left(\left.\frac{d Q}{d P}\right|_{\mathcal{A}_{T}} \frac{S_{t}^{0}}{S_{T}^{0}} H_{T}\right)=E_{t}^{Q}\left(\frac{S_{t}^{0}}{S_{T}^{0}} H_{T}\right)
$$

which demonstrates agreement between the real-world and risk neutral valuation formulae in this case.

We now consider the valuation of contingent claims which are dependent upon stochastic processes defined on our filtered probability space, independent of $\mathbf{W}$ and which are not traded in our financial market. In particular, when the mortality rate is independent of the GOP, the real world pricing formula values a mortality-linked payoff $H_{T}$, which is independent of the GOP and paid at future time $T$, as

$$
V_{t}=E_{t}\left(\frac{S_{t}}{S_{T}} H_{T}\right)=E_{t}\left(\frac{S_{t}}{S_{T}}\right) E_{t}\left(H_{T}\right)=P(t, T) E_{t}\left(H_{T}\right),
$$

where $P(t, T)$ is the real world value at time $t$ of a $T$-maturity zero-coupon bond. Here, the connection with actuarial pricing or with the calculation of net present value is made, as described in Section 10.4 of Platen and Heath [2006]. 


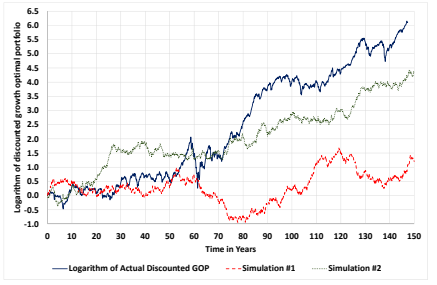

(A) Black-Scholes model.

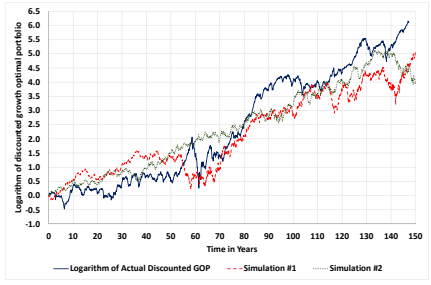

(B) Minimal market model.

FiguRE 5. Comparison of the logarithm of the actual discounted stock index with two sets of simulations of the logarithm of the discounted stock index over 150 years under each of the BlackScholes and minimal market models, commencing at January 1871 and using the parameters in Table 2 .

\section{Appendix B. Simulations of Models}

We provide in Figures 5, 6, 7 and 8 graphs of some Monte Carlo simulations of the stochastic processes for the discounted GOP $\bar{S}_{t}$, short rate $r_{t}$ and mortality rate $\mu_{x+t}(t)$, based on the fitted parameters in Tables 2, 3 and 5. Alongside the simulations are shown the actual behaviors of the market variables for comparison and to check the appropriateness of the models. Although two simulations of each of the models do not provide a complete picture of the multitudes of paths, they do allow for some comparison with actual behaviour and provide at least some idea of the possible scenarios under the models.

In Figure 5 we see that simulations of the BS model and MMM appear to generate discounted GOP paths in line with actual market behaviour. In Figure 6 we see that simulations of the Vasicek and CIR models appear to generate paths of the short rate in line with actual market behaviour. However, Plot (C) within Figure 6 highlights the potentially explosive behaviour of the short rate under the $3 / 2$ model.

In Figures 7 and 8 the simulations of mortality rates deviate substantially from actual rates, as mentioned earlier at the end of Subsection 4.1 and supported by graphs of survival probabilities in Figures 1 and 2.

\section{Appendix C. Simulated Reserves and Policy Values Under Different AsSUMPTIONS}

Our assumptions for the management fee $\xi$, roll-up rate $g$ for the guarantee, factor of $f=0.5$ applied to $g$ for the roll-up rate in respect of the surrender benefit, and the lapse rate $\rho$ are varied in this section to illustrate how these affect the reserves and policy values throughout the life of the policy portfolio.

In Figure 9 we show the reserves and policy values under both the MMM and Black-Scholes model and based on random walk mortality and Vasicek short rate, varying the management fee $\xi$ and roll-up rate $g$ over values in the set $\{0.02,0.03,0.04\}$. 


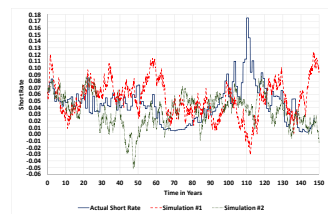

(A) Vasicek model.

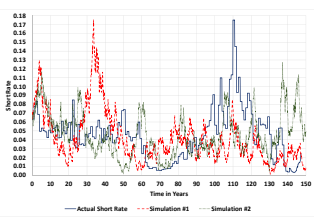

(B) CIR model.

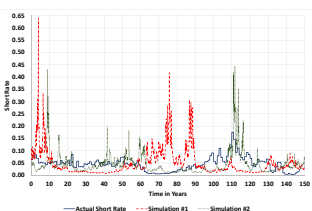

(c) $3 / 2$ model.

Figure 6. Comparison of actual short rates with two sets of simulations of the short rate over 150 years under each of the Vasicek, CIR and 3/2 models, commencing at January 1871 and using the parameters in Table 3 .

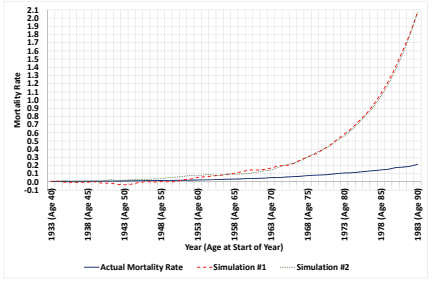

(A) GMSRW model.

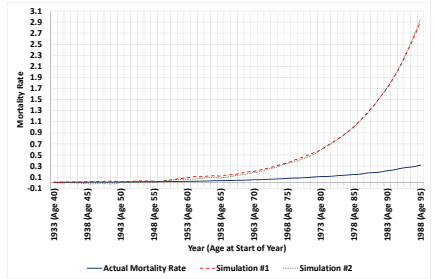

(в) GMSOU model.

FIGURE 7. Comparison of the actual mortality rates with two sets of simulations of mortality rates in respect of a male life aged 40 in 1933, under each of the GMSRW and GMSOU models, having the parameters in Table 5 .

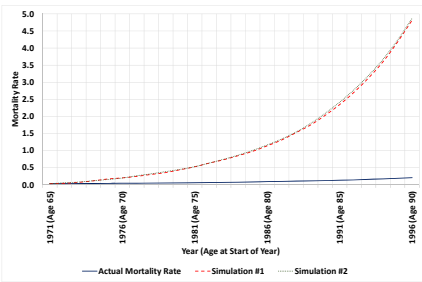

(A) GMSRW model.

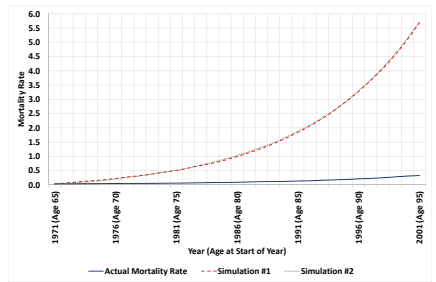

(в) GMSOU model.

Figure 8. Comparison of the actual mortality rates with two sets of simulations of mortality rates in respect of a male life aged 65 in 1971, under each of the GMSRW and GMSOU models, having the parameters in Table 5. 


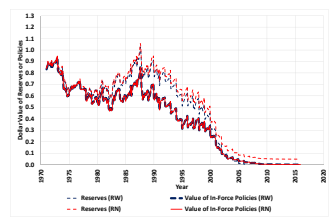

(A) $\xi=0.02$ and $g=0.02$.

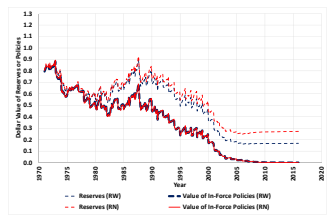

(D) $\xi=0.03$ and $g=0.02$.

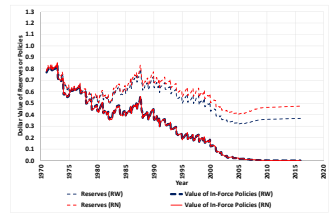

(G) $\xi=0.04$ and $g=0.02$.

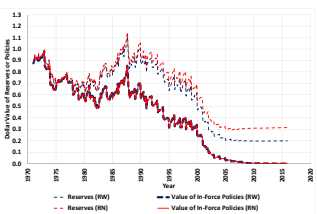

(B) $\xi=0.02$ and $g=0.03$.

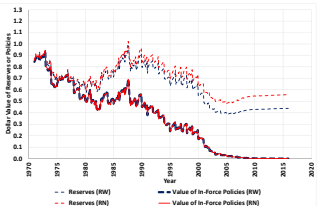

(E) $\xi=0.03$ and $g=0.03$.

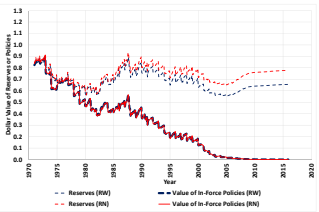

(н) $\xi=0.04$ and $g=0.03$.

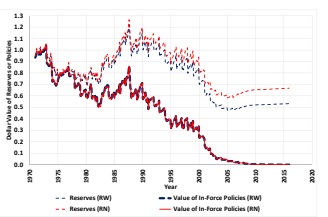

(C) $\xi=0.02$ and $g=0.04$.

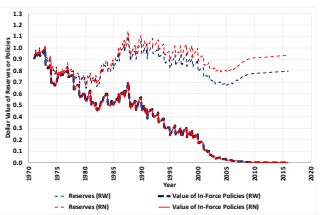

(F) $\xi=0.03$ and $g=0.04$.

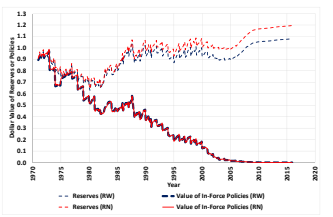

(I) $\xi=0.04$ and $g=0.04$.

Figure 9. Comparison of reserves and policy values, under both the MMM and Black-Scholes model and based on random walk mortality and Vasicek short rate, for various values of $\xi$ and $g$, while maintaining $f=0.5$ and $\rho=0.03$.

In Figure 10 we show the reserves and policy values under both the MMM and Black-Scholes model, based on random walk mortality and Vasicek short rate, varying the lapse rate $\rho$ over values in the set $\{0.02,0.03,0.04\}$.

In Figure 11 we show the reserves and policy values under both the MMM and Black-Scholes model, based on random walk mortality and Vasicek short rate, varying the factor $f$, which is applied to the roll-up rate $g$ for computing the surrender benefit, over values in the set $\{0.25,0.5,0.75\}$.

As also illustrated in Figure 3, Figures 9, 10 and 11 illustrate the policy values and reserving strategies under the BS and MMM models, with random walk mortality and Vasicek short rate, where the surplus levels of reserves attained by the strategy in respect of the MMM are evident in the latter years of the policy, despite having lower initial reserves. Furthermore, these surpluses generated via the MMM 


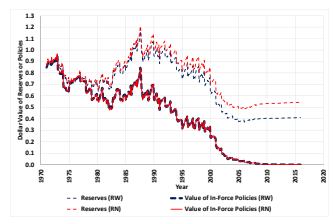

(A) $\rho=0.02$.

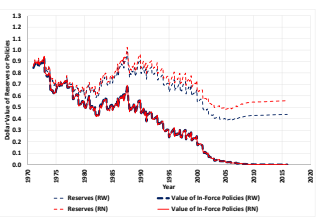

(B) $\rho=0.03$.

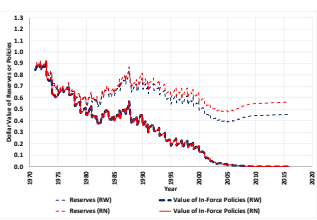

(C) $\rho=0.04$.

Figure 10. Comparison of reserves and policy values, under both the MMM and Black-Scholes model and based on random walk mortality and Vasicek short rate, for various values of $\rho$, while maintaining $\xi=0.03, g=0.03$ and $f=0.5$.

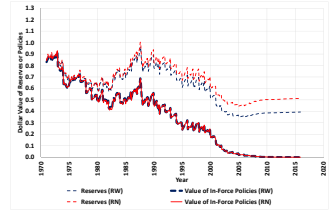

(A) $f=0.25$.

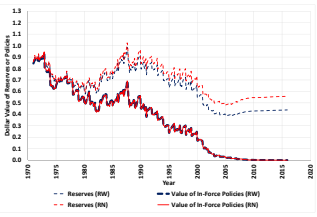

(в) $f=0.5$.

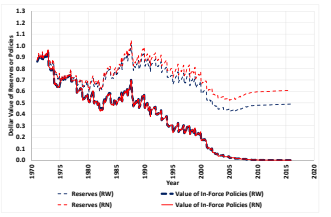

(C) $\rho=0.75$.

Figure 11. Comparison of reserves and policy values, under both the MMM and Black-Scholes model and based on random walk mortality and Vasicek short rate, for various values of $f$, while maintaining $\xi=0.03, g=0.03$ and $\rho=0.03$.

strategy are helpful when actual mortality and lapse rates vary from those assumed in our models, and demonstrate the less-expensive reserving method proposed.

Current address, Kevin Fergusson: University of Melbourne

Victoria 3010, Australia

Email address, Kevin Fergusson: kevin.fergusson@unimelb.edu.au

$U R L:$ http://fbe.unimelb.edu.au/economics/ACT 